ARTICLE

\title{
Targeted apoptosis of macrophages and osteoclasts in arthritic joints is effective against advanced inflammatory arthritis
}

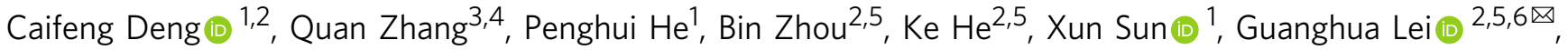 \\ Tao Gong (iD) ${ }^{1 \otimes} \&$ Zhirong Zhang ${ }^{1}$
}

Insufficient apoptosis of inflammatory macrophages and osteoclasts (OCs) in rheumatoid arthritis (RA) joints contributes toward the persistent progression of joint inflammation and destruction. Here, we deliver celastrol (CEL) to selectively induce apoptosis of OCs and macrophages in arthritic joints, with enzyme-responsive nanoparticles (termed PRNPs) composed of RGD modified nanoparticles (termed RNPs) covered with cleavable PEG chains. CEL-loaded PRNPs (CEL-PRNPs) dually target OCs and inflammatory macrophages derived from patients with RA via an RGD- $\alpha v \beta 3$ integrin interaction after PEG cleavage by matrix metalloprotease 9, leading to increased apoptosis of these cells. In an adjuvant-induced arthritis rat model, PRNPs have an arthritic joint-specific distribution and CEL-PRNPs efficiently reduce the number of OCs and inflammatory macrophages within these joints. Additionally, rats with advanced arthritis go into inflammatory remission with bone erosion repair and negligible side effects after CEL-PRNPs treatment. These findings indicate potential for targeting chemotherapy-induced apoptosis in the treatment of advanced inflammatory arthritis.

\footnotetext{
${ }^{1}$ Key Laboratory of Drug-Targeting and Drug Delivery System of the Education Ministry, Sichuan Engineering Laboratory for Plant-Sourced Drug and Sichuan Research Center for Drug Precision Industrial Technology, West China School of Pharmacy, Sichuan University, Chengdu 610064, China. ${ }^{2}$ Department of Orthopaedics, Xiangya Hospital, Central South University, Changsha 410008, China. ${ }^{3}$ Institute of Materia Medica, School of Pharmacy, Chengdu Medical College, Chengdu 610500, China. ${ }^{4}$ Development and Regeneration Key Lab of Sichuan Province, Department of Pathology, Department of Anatomy and Histology and Embryology, Chengdu Medical College, Chengdu 610500, China. ${ }^{5}$ Hunan Key Laboratory of Joint Degeneration and Injury, Changsha 410008 , China. ${ }^{6}$ National Clinical Research Center of Geriatric Disorders, Xiangya Hospital, Central South University, Changsha 410008, China.

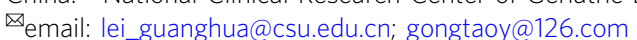


R heumatoid arthritis (RA) is a chronic autoimmune inflammatory disease characterized by synovial inflammation and joint destruction ${ }^{1}$. Synovial inflammation is the dominant feature in the early stage of $\mathrm{RA}^{2-4}$. In addition to the inflammatory symptoms of joint swelling and synovial inflammation, obvious cartilage damages and bone erosion occur during the persistent progression of RA, which can eventually result in joint deformity and disability ${ }^{5-7}$. Patients with advanced RA suffer from the loss of physical function and low quality of life ${ }^{7,8}$. Therefore, a rational therapeutic approach that can alleviate synovial inflammation and reverse bone erosion is urgently needed for the treatment of advanced RA.

Macrophages and osteoclasts (OCs) have been demonstrated to play key roles in the pathogenesis of RA. The increase in the abundance of synovial macrophages is an early hallmark of rheumatic disease. Synovial macrophages from RA patients show distinct activation states and represent one potential key mediator of joint inflammation ${ }^{3,9,10}$. Additionally, studies have revealed large numbers of OCs at sites of arthritic bone erosion ${ }^{11-13}$. OCs are terminally differentiated cells with the unique ability to resorb bone matrix ${ }^{14-16}$. Notably, OCs in RA-affected joints can accelerate synovial inflammation through the production of proinflammatory cytokines ${ }^{17,18}$. Both OCs and synovial inflammatory macrophages express high levels of av $\beta 3$ integrin. av $\beta 3$ integrin has been demonstrated to play important role in activated macrophage-dependent inflammation and OC-dependent bone resorption ${ }^{19,20}$. Generally, the life spans of macrophages and OCs are precisely regulated by apoptosis to maintain immune homeostasis and bone function balance, respectively ${ }^{21-24}$. However, macrophages and OCs from RA joints show decreased apoptotic rates compared with those from healthy controls ${ }^{21,25,26}$. The insufficient apoptosis of macrophages and OCs in the RA joint contributes toward the persistent progression of joint inflammation and joint destruction. Accordingly, inducing the apoptosis of both macrophages and OCs in RA joints is a promising strategy for advanced RA therapy.

Current antirheumatic drugs, including glucocorticoids and biological antibodies, mainly target the macrophages-induced inflammatory response to reduce synovial inflammation ${ }^{5,27}$. However, the application of glucocorticoids can result in severe side effects including bone loss and hyperglycemia ${ }^{28,29}$, while the frequent use of antibodies to block inflammatory cytokines can cause systemic immune suppression, thereby leading to a high risk of infections ${ }^{30,31}$. Furthermore, not all patients respond to antibody therapy and the benefits of using antibody therapy is short-lived ${ }^{21,30,32}$. Recently, Janus Kinase (JAK) inhibitors have been reported to have the potential to reverse the bone erosions in $\mathrm{RA}^{33,34}$, yet JAK inhibition could lead to serious and opportunistic infections ${ }^{33,35}$. Celastrol (CEL), a cytotoxic chemotherapeutic drug, can induce apoptosis in tumor cells and has been widely studied in cancer therapy ${ }^{36,37}$. In addition to its known efficacy in cancer treatment, CEL was previously shown in our laboratory to also treat glomerulonephritis by inducing the apoptosis of mesangial cells ${ }^{38}$. In a rat model of anti-Thy1.1 nephritis, the targeted delivery of CEL to the mesangial cells significantly increased its therapeutic efficacy and decreased its side effects ${ }^{38}$. Inspired by our previous findings, we hypothesized that selectively delivering CEL to both macrophages and OCs in RA joints may efficiently induce apoptosis in these cells, thus reducing synovial inflammation and reversing bone erosion in advanced RA.

In this study, we report the development of matrix metalloproteinase 9 (MMP9)-cleavable, polyethylene glycol (PEG)- and RGD peptide-modified poly (D, L-lactide-co-glycolide) (PLGA) nanoparticles (termed "PRNPs") for the targeted delivery of CEL to both OCs and macrophages in arthritic joints. PRNPs show high cellular uptake in both OCs and inflammatory macrophages derived from patients with late-stage RA via RGD- $\alpha v \beta 3$ integrin interaction after responding to MMP9. In addition, CEL-loaded PRNPs (CEL-PRNPs) in the presence of MMP9 effectively induce the apoptosis of these cells. In adjuvant-induced arthritis (AIA) rats, PRNPs show an arthritic joints-specific distribution and target both OCs and macrophages within these joints. Further, the intravenous administration of CEL-PRNPs effectively relieves the ankle and paw swelling, restores the balance of bone function, and reverses bone erosion in the inflamed joints of AIA rats with advanced arthritis. Of note, CEL-PRNPs seem safe and induce negligible apoptosis in normal organs. In summary, CEL-PRNPs show great promise in promoting inflammatory remission and bone erosion repair in advanced inflammatory arthritis.

\section{Results}

Features of pathology in different stages of arthritis. AIA in the rat is a well-characterized model for assessing the stages of pathology in RA and exploring arthritis mechanisms ${ }^{39-41}$. Using micro-computed tomography (micro-CT) and histological assays, we investigated joint destruction and synovial inflammation in AIA rats. Micro-CT was used to scan the ankle joints of both normal rats and AIA rats. We found that, in contrast with the smooth bone surface and negligible bone erosion in ankle joints from rats with early-stage arthritis, AIA rats with advanced arthritis displayed rough bone surfaces, severe bone erosion, and significantly decreased bone mineral density (BMD) (Fig. 1a-c). Applying tartrate-resistant acid phosphatase (TRAP) staining of OCs further demonstrated that there were large numbers of OCs and areas of bone erosion in AIA rats within the advanced arthritis group (Fig. 1a). We also examined cartilage integrity using safranin $\mathrm{O}$ and toluidine blue staining to label the glycosaminoglycans (GAGs) in cartilage tissues ${ }^{42,43}$. The cartilage of AIA rats with early-stage arthritis remained intact and was comparable to that of the normal group. However, significant reductions of safranin $\mathrm{O}$ - and toluidine blue-positive areas were observed on the cartilage surface of AIA rats with advanced arthritis, indicating that a loss of GAGs and cartilage destruction occurred in advanced arthritis (Supplementary Fig. 1). We next investigated levels of synovial inflammation in AIA rats using immunohistochemical staining. Synovial macrophages with high potential to induce inflammation were identified as $\mathrm{CD}^{+} 8^{+}$ cells ${ }^{44-46}$. Immunohistochemical images revealed that rats with disease induction showed increased infiltration of $\mathrm{CD}^{+} 8^{+}$macrophages into the synovium compared with the normal group (Fig. 1a). Notably, AIA rats with advanced arthritis showed an excessive abundance of $\mathrm{CD}^{+} 8^{+}$macrophages and synovial hyperplasia, consistent with the results of hematoxylin and eosin (H\&E) assay (Fig. 1a; Supplementary Fig. 1). In addition to the increased accumulation of $\mathrm{CD}^{+} 8^{+}$macrophages, the augmented expression of MMP9 in the arthritic inflammatory environment was also observed during the induction of AIA (Fig. 1a). Previous studies also demonstrated macrophages and OCs promoted the high expression of MMP9 in the inflammatory microenvironment of $\mathrm{RA}^{47,48}$. Thus, these results revealed that there were excessive levels of $\mathrm{CD}^{+} 8^{+}$macrophages and OCs in the arthritic joints of AIA rats with advanced arthritis.

Preparation and characterization of CEL-PRNPs. PLGA nanoparticles exhibit good biocompatibility and high drug loading efficacy and have been widely employed as a drug delivery system ${ }^{49-51}$. Therefore, PLGA nanoparticles were adopted as the drug carrier in this work. We first modified PLGA with RGD peptide via the maleimide-thiol coupling reaction between CysRGD and Mal-PEG-PLGA and the chemical structure was 


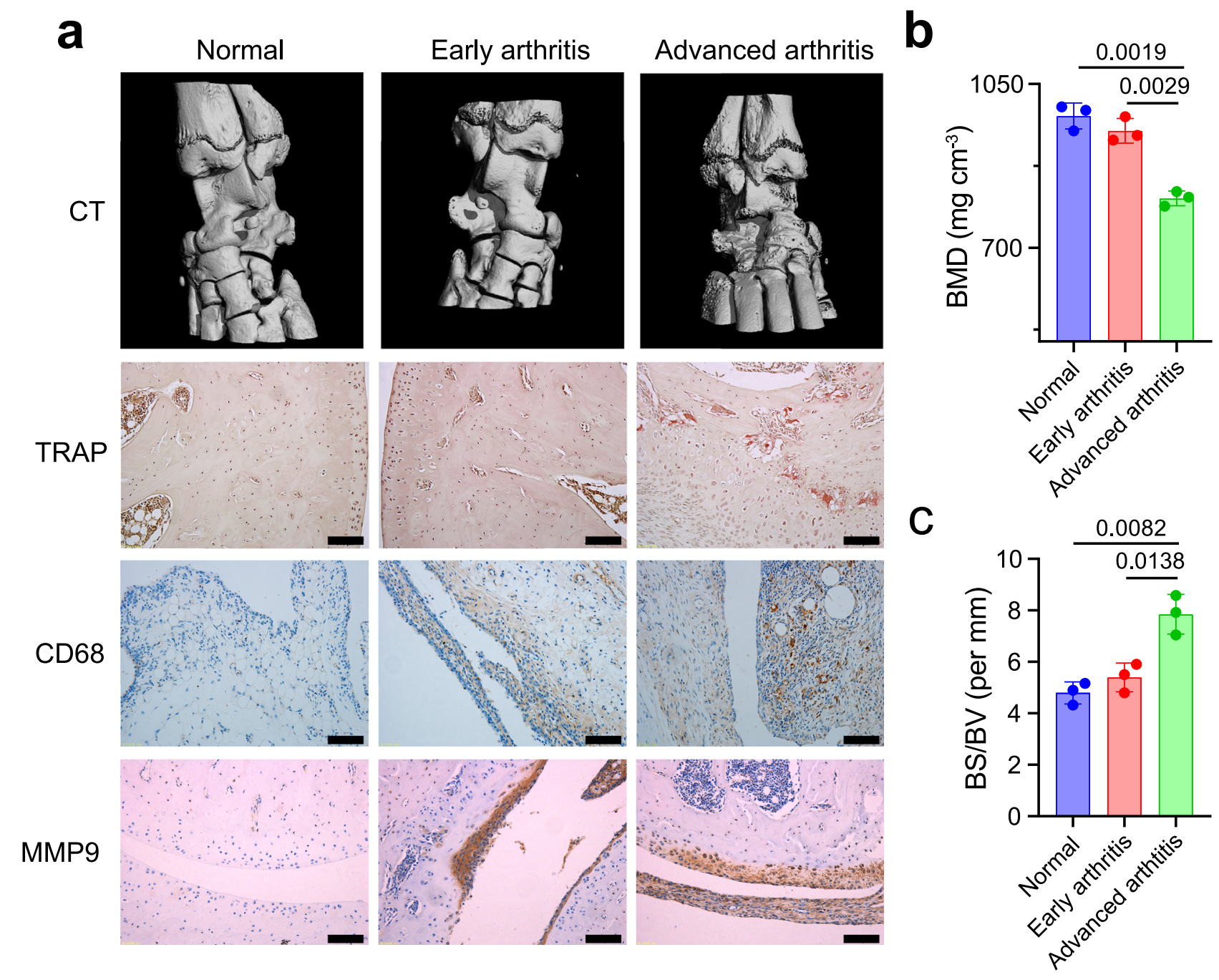

Fig. 1 Pathological features differ between rats with early and advanced arthritis. a OCs-induced bone erosion and macrophages-mediated synovial inflammation in advanced arthritis. Representative micro-CT images of ankle joints showing bone erosion levels in ankle joints from normal rats, AIA rats with early-stage arthritis, and AIA rats with late-stage arthritis. Immunohistochemical analyses of the TRAP (tartrate-resistant acid phosphatase)-stained OCs, CD68+ synovial macrophages, and MMP9 (matrix metalloproteinase 9 ) expression in the joint tissues from rats in each group $(n=3)$. Scale bar $=$ $100 \mu \mathrm{m}$. b, c Quantitative micro-CT analyses of bone mineral density (BMD) and bone surface density (BS/BV). Data represent the mean \pm SD ( $n=3$ independent animals). Statistical significance was determined by a two-sided Student's $t$ test.

analyzed by proton nuclear magnetic resonance $\left({ }^{1} \mathrm{H}-\mathrm{NMR}\right)$ spectroscopy (Supplementary Fig. 2). Different PLGA nanoparticles were prepared as shown in Fig. 2a. Briefly, CEL-loaded PLGA nanoparticles (CEL-NPs) and CEL-loaded, RGD-modified PLGA nanoparticles (CEL-RNPs) were both prepared using an emulsion/solvent evaporation method. To obtain CEL-PRNPs, $\mathrm{PEG}_{2000}$-MMP9 cleavable peptide was linked to CEL-RNPs using the water phase reaction method ${ }^{52}$. The particle sizes of CEL-NPs and CEL-RNPs were $155.7 \pm 4.9 \mathrm{~nm}$ and $154.1 \pm 4.6 \mathrm{~nm}$, respectively. After covered with cleavable $\mathrm{PEG}_{2000}$, the particle size of CEL-PRNPs slightly increased to $162.2 \pm 6.6 \mathrm{~nm}$ (Fig. 2b; Supplementary Table 1 ). The average zeta potentials of CEL-RNPs and CEL-PRNPs were $-3.2 \pm 0.6 \mathrm{mV}$ and $-5.3 \pm 0.4 \mathrm{mV}$, respectively. The CEL encapsulation efficiencies of various developed PLGA nanoparticles were all close to 90\% (Supplementary Table 1). The transmission electron microscope (TEM) images demonstrated that the morphologies of the prepared CELPRNPs were generally spherical and uniformly dispersed (Fig. 2c). The serum stability assay was conducted to investigate interactions between various developed PLGA nanocarriers and blood components. The average particle sizes remained nearly unchanged for CEL-NPs, CEL-RNPs, and CEL-PRNPs after $24 \mathrm{~h}$ of storage in $10 \%$ fetal bovine serum (FBS) at $37^{\circ} \mathrm{C}$ (Fig. 2d; Supplementary Fig. 3), suggesting the good stability of these nanoparticles in serum.

CEL-PRNPs increase apoptosis of OCs and inflammatory macrophages. To investigate whether PRNPs could target OCs and pathogenic macrophages via RGD-mediated endocytosis, a cellular uptake study was performed. OCs were established by using M-CSF- and RANKL-stimulated bone marrow macrophages (BMMs) and pathogenic macrophages were obtained by using lipopolysaccharide (LPS)-activated BMMs ${ }^{53}$. The successful genesis of OCs was confirmed using TRAP staining. Results showed that RANKL-induced OCs stained red and were multinuclear, while the unstimulated BMMs stained yellow (Supplementary Fig. 4), suggesting that OCs were efficiently generated via RANKL stimulation. To investigate the distribution behaviors of various prepared nanoparticles in OCs and LPS-activated macrophages, the fluorescent probe, coumarin 6 (C6), was loaded into the nanoparticles. Confocal images of the OCs and LPSactivated macrophages showed that RNPs conferred significantly 

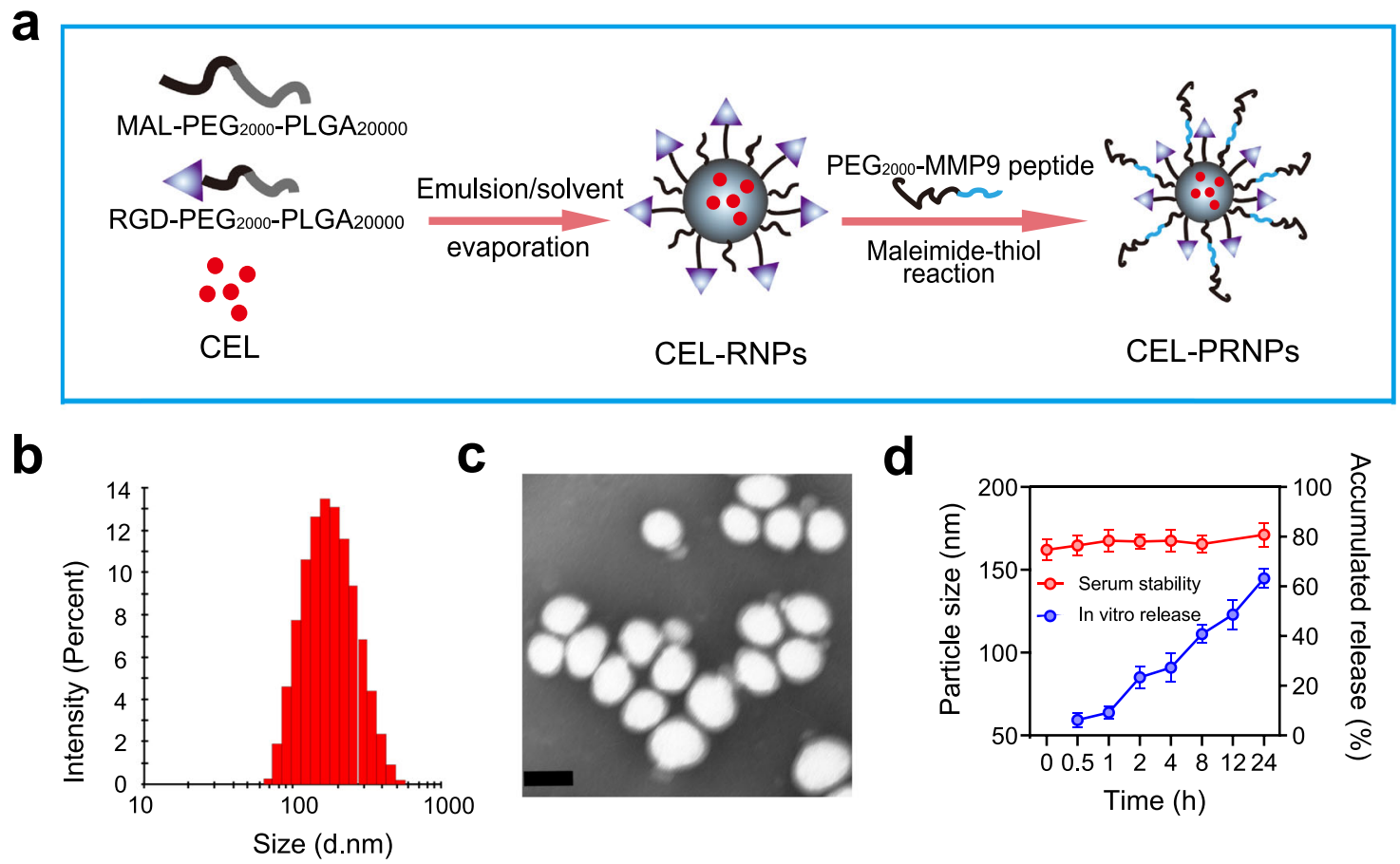

Fig. 2 Preparation and characterization of CEL-PRNPs. a Schematic illustration of CEL-PRNPs preparation. CEL celastrol, MMP9 matrix metalloproteinase 9, CEL-RNPs CEL-loaded RGD peptide-modified poly (D, L-lactide-co-glycolide) (PLGA) nanoparticles, CEL-PRNPs CEL-loaded MMP9-cleavable polyethylene glycol (PEG)- and RGD peptide-modified PLGA nanoparticles. b Representative size distribution image of CEL-PRNPs ( $n=3$ independent samples). c TEM image of CEL-PRNPs ( $n=3$ independent samples). Scale bar $=100 \mathrm{~nm}$. $\mathbf{d}$ The serum stability of CEL-PRNPs during $24 \mathrm{~h}$ incubation with $10 \% \mathrm{FBS}$ at $37^{\circ} \mathrm{C}$ and cumulative CEL release from CEL-PRNPs in PBS at $37^{\circ} \mathrm{C}$. Data represent mean \pm SD $(n=3$ independent samples).

increased green fluorescence signal to these cells compared with NPs (Fig. 3a; Supplementary Figs. 5 and 6). However, this effect was not observed in the non-activated BMMs (Supplementary Fig. 7). These results suggested that the RNPs could selectively target both OCs and LPS-activated macrophages for cellular uptake. In the absence of MMP9, PRNPs showed remarkably reduced cellular uptake, whereas, in the presence of MMP9, this effect was reversed (Fig. 3a; Supplementary Figs. 5 and 6). This indicated that the PEG chains on the PRNPs could be cleaved by MMP9, thereby exposing the RGD peptide for the selective targeting of the OCs and LPS-activated macrophages. The results of quantitative cellular uptake analysis based on flow cytometry showed the same trends (Fig. 3d, e). Furthermore, the dualtargeting ability of PRNPs was also investigated on human OCs and inflammatory macrophages. Human OCs were obtained by using M-CSF- and RANKL-stimulated peripheral blood mononuclear cells from patients with late-stage RA. Human inflammatory macrophages were isolated by magnetic-activated cell sorting method from synovial tissues of patients with late-stage RA undergoing joint replacement surgery. As shown in Fig. 4a-c, the increased distribution of RNPs and PRNPs (in the presence of MMP9) were also observed on OCs and inflammatory macrophages derived from patients with late-stage RA. These results demonstrated that RNPs and PRNPs (in the presence of MMP9) could target both OCs and inflammatory macrophages through ligand-receptor interactions.

The inadequate apoptosis of macrophages and OCs in the rheumatoid inflammatory microenvironment is an important pathomechanism in synovial hyperplasia and joint destruction $^{21,54,55}$. To investigate whether CEL-PRNPs could effectively induce the apoptosis of OCs and inflammatory macrophages, their apoptotic profile was determined by flow cytometry assay. As shown in Fig. 3b, f, g, CEL-RNPs treatment significantly increased the apoptotic cell percentages in both OCs and LPS-activated macrophages compared with the CEL-NPs and CEL-PRNPs. However, in the presence of MMP9, treatment with the CEL-PRNPs resulted in high levels of apoptosis in both OCs and LPS-activated macrophages, demonstrating that CEL-PRNPs were MMP9-responsive. As mitochondrial dysfunction is also a hallmark of apoptosis, we further measured the mitochondrial membrane potential (a marker of mitochondrial dysfunction) in macrophages with the JC- 1 dye ${ }^{56}$. The JC- 1 dye tends to aggregate (with red fluorescence) in normal mitochondria, and its color changes from red to green when the membrane potential collapses $^{57}$. Confocal images showed that both CEL-RNPs and CEL-PRNPs (in the presence of MMP9) resulted in significantly higher green fluorescence intensity signal compared with CELNPs (Fig. 3c), suggesting the severe disruption of the mitochondrial membrane in LPS-activated macrophages. Furthermore, CEL-RNPs and CEL-PRNPs (in the presence of MMP9) also triggered the higher rates of apoptosis among OCs and inflammatory macrophages derived from patients with latestage RA, when compared with CEL-NPs and CEL-PRNPs (in the absence of MMP9) (Fig. 4e, f). These results proved that CELPRNPs had excellent ability to cause the apoptosis of OCs and inflammatory macrophages after responding to MMP9.

PRNPs target both OCs and inflammatory macrophages in arthritic joints. We studied the biodistribution of the PRNPs in AIA rats with advanced arthritis using an in vivo imaging system and PRNPs loaded with DiD, a fluorescent probe. The in vivo fluorescence images showed that PRNPs significantly increased the $\mathrm{DiD}$ fluorescence distribution in inflamed joints compared with NPs and RNPs (Fig. 5a, d; Supplementary Fig. 8). Of note, the use of PRNPs remarkably reduced the DiD fluorescence distribution in the lung, liver and spleen compared with RNPs (Fig. 5a, e), which indicated that the nonspecific distribution of RNPs was avoided through the use of a surface coating 


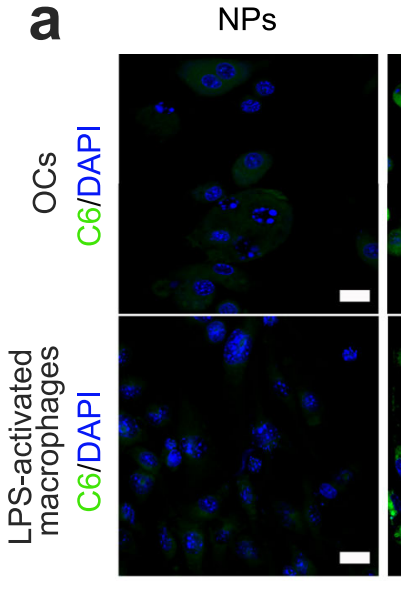

b
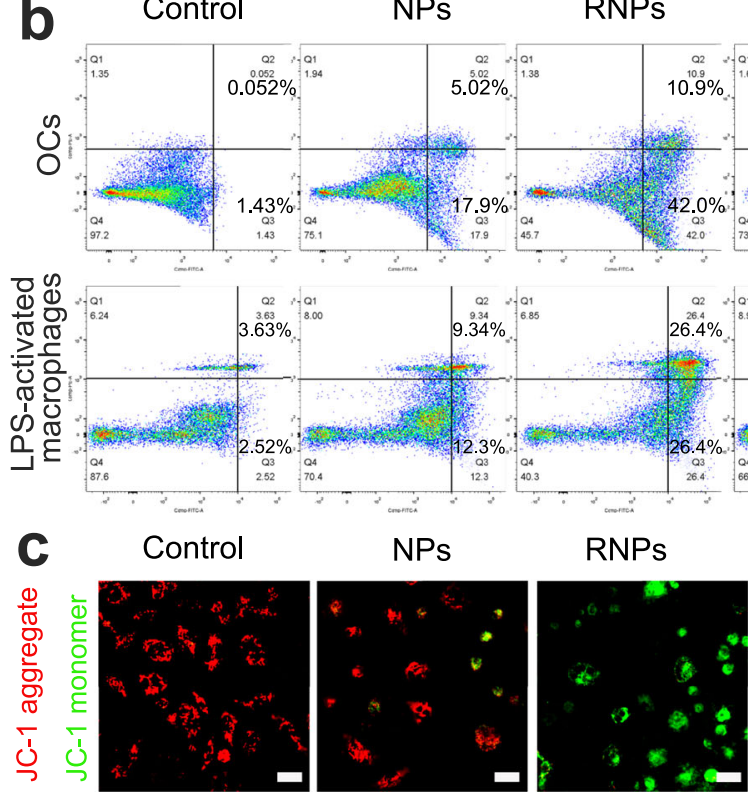

NPs

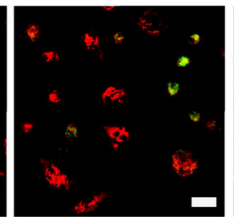

RNPS

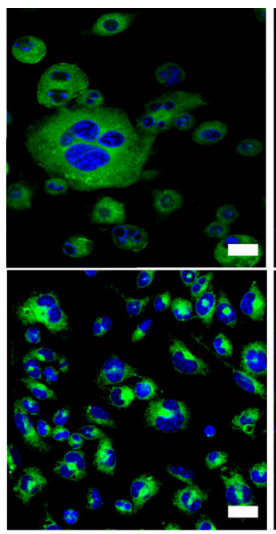

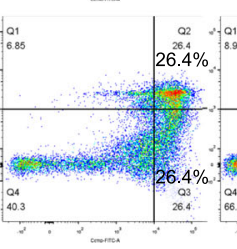

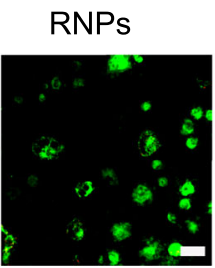

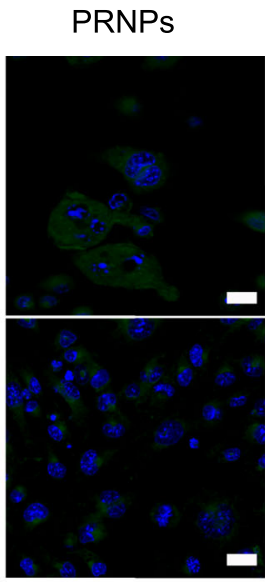

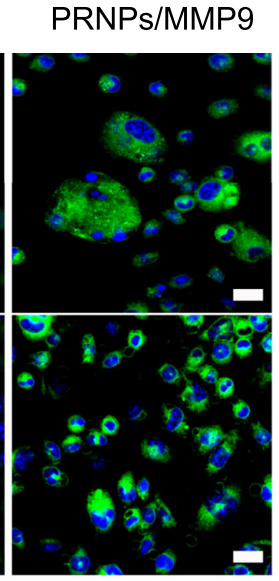

PRNPS

PRNPs/MMP9
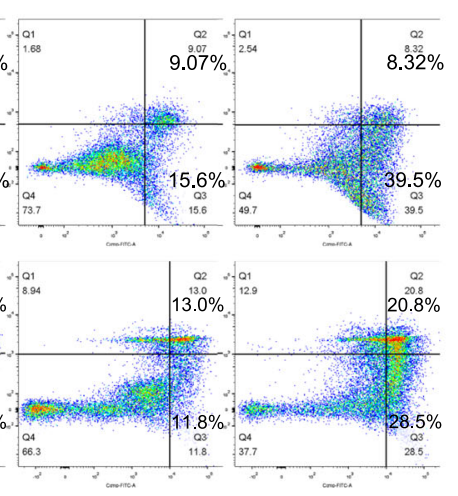

PRNPs
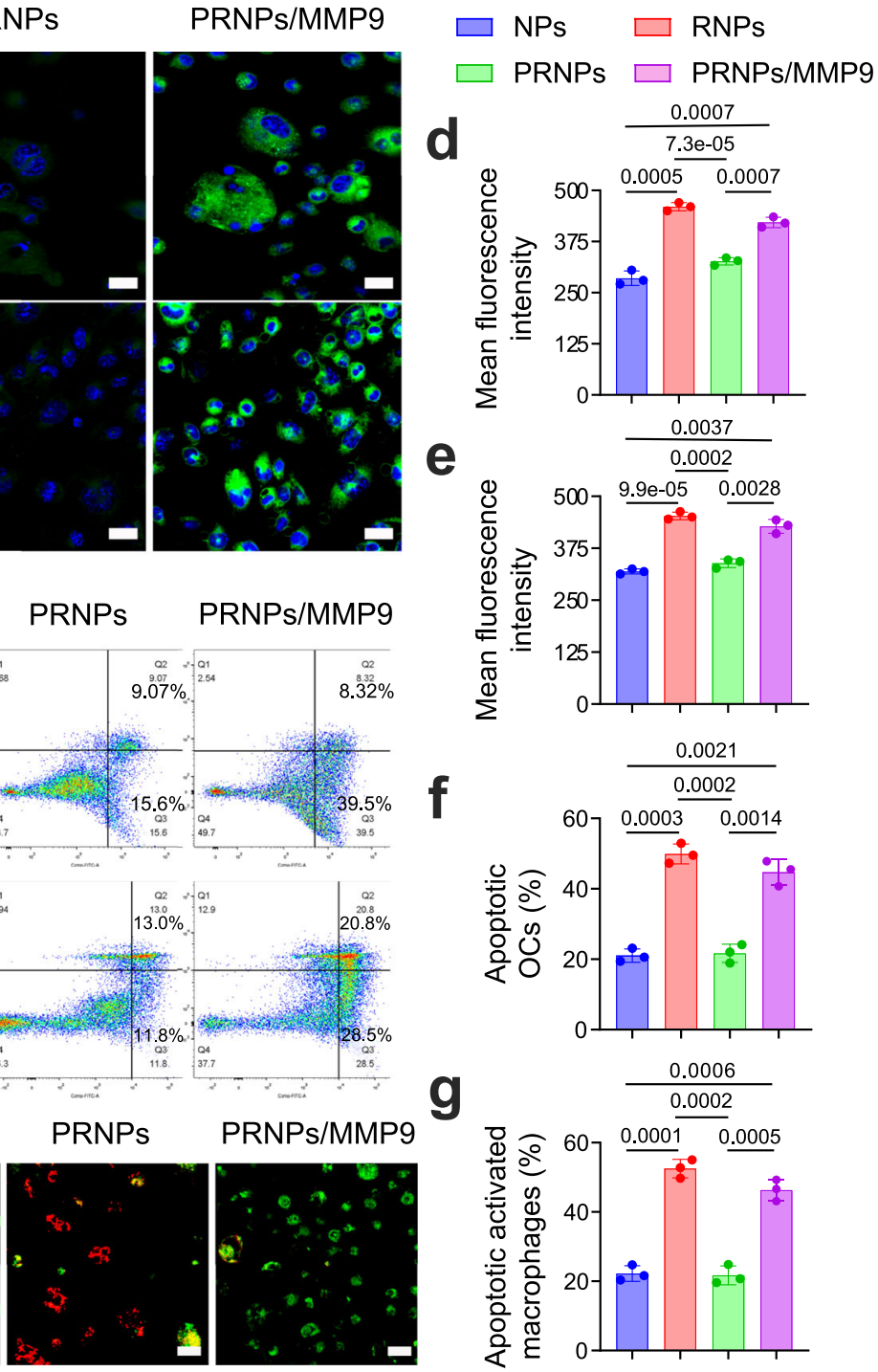

Fig. 3 Increased apoptosis of OCs and LPS-activated macrophages caused by RGD-mediated endocytosis of PRNPs. a Confocal images of cellular uptake in OCs and LPS-activated macrophages ( $n=3$ independent samples). Scale bar $=50 \mu \mathrm{m}$. b Flow cytometric analysis of OCs and LPS-activated macrophages apoptosis induced by CEL-RNPs, CEL-RNPs, or CEL-PRNPs with or without the presence of MMP9 for $24 \mathrm{~h}$ at the CEL concentration of 100 $\mathrm{ng} / \mathrm{mL}$. c Confocal images showing JC-1 assay of LPS-activated macrophages treated with CEL-RNPs, CEL-RNPs, or CEL-PRNPs with or without the presence of MMP9 for $24 \mathrm{~h}$ at the CEL concentration of $100 \mathrm{ng} / \mathrm{mL}$ ( $n=3$ independent samples). Scale bar $=50 \mu \mathrm{m}$. d, e Quantitative cellular uptake C6loaded NPs, C6-loaded RNPs, or C6-loaded PRNPs on OCs (d) and LPS-activated macrophages (e) after $1 \mathrm{~h}$ incubation at the C6 concentration of 50 ng/ $\mathrm{mL}$. Data represent mean \pm SD ( $n=3$ independent samples). Statistical significance was determined by a two-sided Student's $t$ test. $\mathbf{f}, \mathbf{g}$ Quantitative analysis for the apoptosis of OCs (f) and LPS-activated macrophages $(\mathbf{g})$ by CEL-NPs, CEL-RNPs, or CEL-PRNPs. Data represent mean \pm SD $(n=3$ independent samples). Statistical significance was determined by a two-sided Student's $t$ test. OCs osteoclasts, LPS lipopolysaccharide, C6 coumarin 6, DAPI 2-(4-Amidinophenyl)-6-indolecarbamidine dihydrochloride, MMP9 matrix metalloproteinase 9, NPs poly (D, L-lactide-co-glycolide) (PLGA) nanoparticles, RNPs RGD peptide-modified PLGA nanoparticles, PRNPs MMP9-cleavable polyethylene glycol (PEG)- and RGD peptide-modified PLGA nanoparticles.

comprising cleavable PEG chains, as av $\beta 3$ integrins are also highly expressed in normal tissues ${ }^{58,59}$. The ex vivo fluorescence images also demonstrated that PRNPs had the longest blood circulation time (Fig. 5a, e), which further suggested that the $\mathrm{PEG}_{2000}$-MMP9 cleavable peptide had been successfully linked to RNPs. Interestingly, PRNPs accumulation in inflamed joints at $24 \mathrm{~h}$ after intravenous injection was still as high as that in inflamed joints at $2 \mathrm{~h}$ (Supplementary Fig. 8). However, their accumulation in normal organs at $24 \mathrm{~h}$ was obviously less than that at $2 \mathrm{~h}$ (Supplementary Fig. 9). These results illustrated that PRNPs displayed the inflamed joint-targeting ability and extended retention in inflamed joints.
Next, AIA rats with unilateral inflamed joints were adopted to evaluate the selective accumulation of PRNPs in inflamed joints compared with normal joints. As shown in Fig. 5b, c, $f$, there were no significant differences in the distribution of free $\mathrm{DiD}$ between inflamed joints and non-inflamed joints. In contrast, DiD-loaded NPs, RNPs, and PRNPs showed a higher accumulation in inflamed joints than non-inflamed joints. As expected, PRNPs displayed the highest distribution in inflamed joints among the three nanoparticle types, which was consistent with the results obtained for AIA rats with bilateral inflamed joints (Fig. 5a, d).

The above results demonstrated that PRNPs increased drug distribution in arthritic joints. To investigate whether PRNPs could 


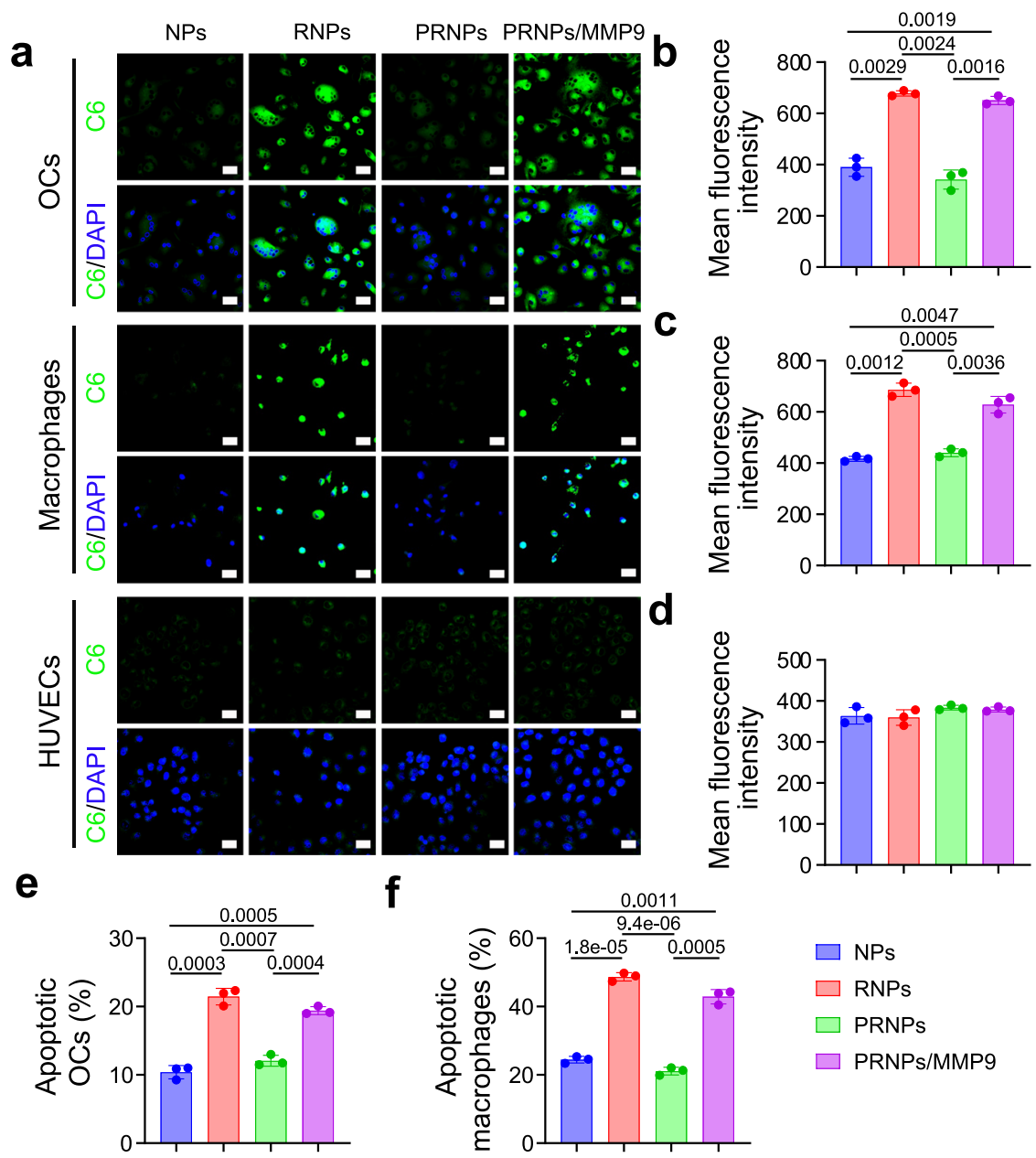

Fig. 4 Increased apoptosis of OCs and synovial macrophages derived from patients with late-stage RA by PRNPs. a Confocal images of the cellular uptake on OCs and synovial macrophages ( $n=3$ independent samples). Scale bar $=50 \mu \mathrm{m}$. b-d Quantitative analysis of the cellular uptake of C6-loaded NPs, RNPs or PRNPs on OCs (b), synovial macrophages (c), and HUVECs (d) after 1-h incubation at the C6 concentration of $50 \mathrm{ng} / \mathrm{mL}$. Data represent mean \pm SD ( $n=3$ independent samples). Statistical significance was determined by a two-sided Student's $t$ test. e, $\mathbf{f}$ Quantitative analysis for the apoptosis of OCs (e) and synovial macrophages (f) by CEL-NPs, CEL-RNPs, or CEL-PRNPs. Data represent mean \pm SD ( $n=3$ independent samples). Statistical significance was determined by a two-sided Student's $t$ test. OCs osteoclasts, C6 coumarin 6, DAPI 2-(4-Amidinophenyl)-6-indolecarbamidine dihydrochloride, MMP9 matrix metalloproteinase 9, HUVECs human umbilical vein endothelial cells, NPs poly (D, L-lactide-co-glycolide) (PLGA) nanoparticles, RNPs RGD peptide-modified PLGA nanoparticles, PRNPs MMP9-cleavable polyethylene glycol (PEG)- and RGD peptide-modified PLGA nanoparticles.

target both OCs and inflammatory macrophages in arthritic joints, the distribution of different $\mathrm{DiD}$ formulations in both types of cells was determined using the immunofluorescent staining method. Inflammatory macrophages and OCs were determined by immunofluorescence analysis of CD68 and CD51 (green fluorescence). As shown in Fig. 6, the DiD fluorescence distribution of PRNPs in the synovial joint was the highest among the three nanoparticle types, which was consistent with the results of in vivo and ex vivo imaging studies. In addition, DiD solution and DiD labeled NPs showed low levels of colocalization of the red (DiD) and green fluorescence, suggesting the nonspecific distributions in inflamed joints. Whereas, the DiD fluorescence of RNPs and PRNPs was mainly overlapped with the green fluorescence in synovial tissues. Therefore, the results proved that PRNPs could detach their PEG chains and transform into RNPs within inflamed joints, so as to efficiently and selectively deliver drugs into OCs and macrophages located in inflamed joints.

CEL-PRNPs decrease the number of OCs and macrophages in arthritic joints. The above results showed that PRNPs exhibited good selectivity toward both OCs and inflammatory macrophages in arthritic joints. Furthermore, CEL-PRNPs (in the presence of MMP9) could more effectively induce apoptosis in both OCs and inflammatory macrophages due to the increasing cellular uptake. To demonstrate that PRNPs could realize the selective reduction of OCs and synovial macrophages at sites of inflammation in AIA rats with advanced arthritis, we measured the level of cellular apoptosis in the inflamed joints, the number of OCs and macrophages in the synovial joints, and the cytokine profiles in blood and joints.

AIA rats with advanced arthritis were randomly divided into five groups based on their treatment with either saline, CEL solution, CEL-NPs, CEL-RNPs, or CEL-PRNPs. Saline or $1 \mathrm{mg} / \mathrm{kg}$ CEL equivalents of CEL solution, CEL-NPs, CEL-RNPs, or CELPRNPs were intravenously injected into rats every other 2 days. Ankle joints were collected for TUNEL staining two days after the last treatment. Inflammatory macrophages and OCs were determined by immunofluorescence analysis of CD68 and CD51 (red fluorescence). As shown in Fig. 7a and Supplementary Fig. 10, free CEL solution led to a very low level of apoptosis in inflamed joints, whereas CEL-PRNPs triggered the highest level 
a

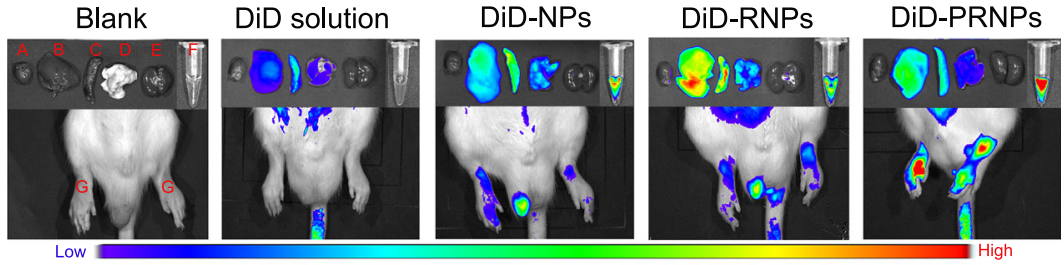

b

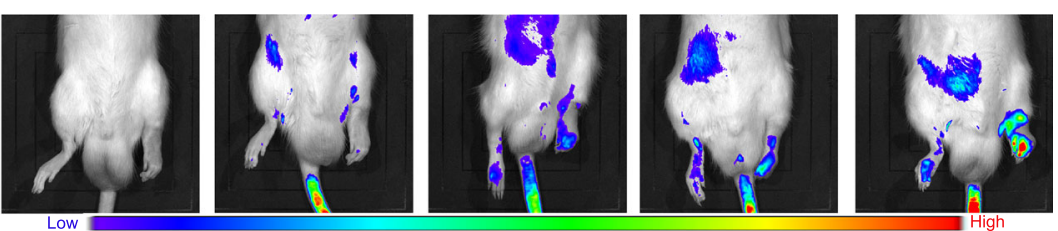

C

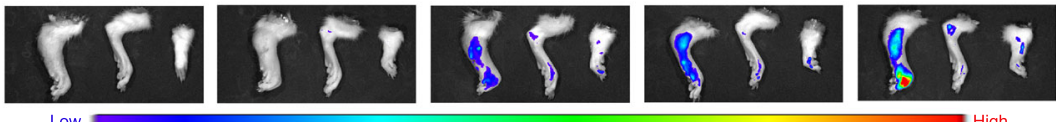

d
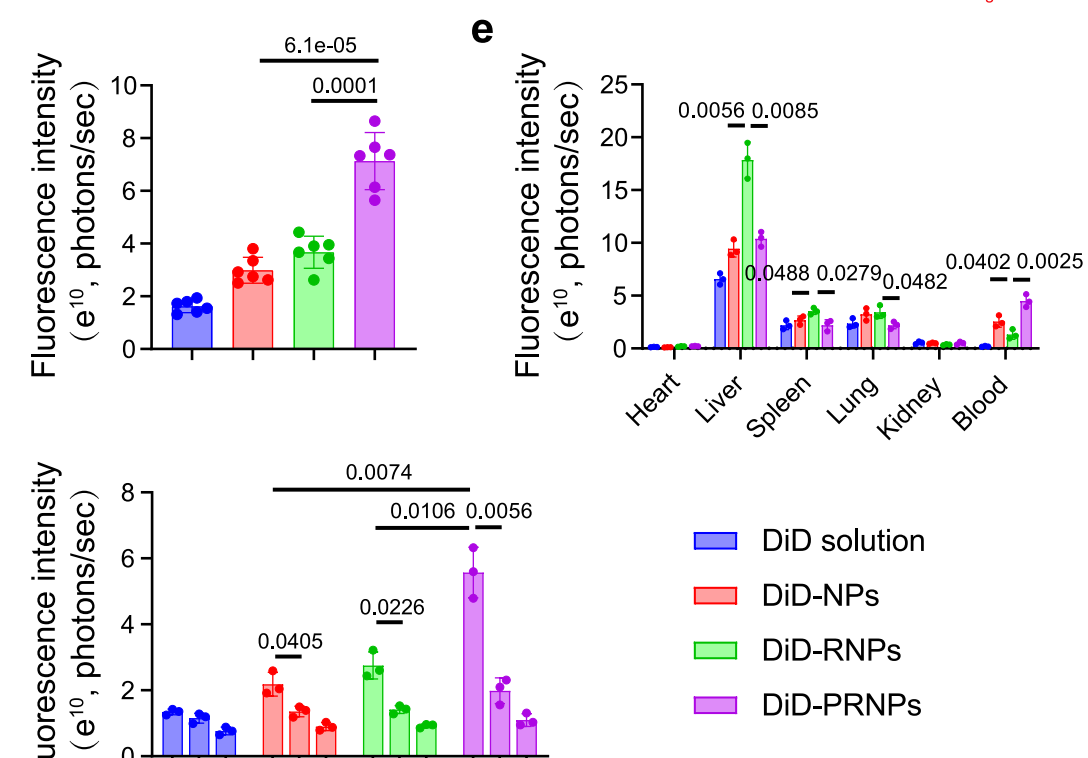

Fig. 5 PRNPs selectively accumulate in inflamed joints of rats with advanced arthritis. a Ex vivo DiD fluorescence images showing the biodistribution of NPs, RNPs, and PRNPs in AIA rats with advanced arthritis (A, heart; B, liver; C, spleen; D, lung; E, kidney; F, Blood; G, arthritic joint) at $24 \mathrm{~h}$ post injection. b In vivo DiD fluorescence images showing the arthritic joint distribution of free DiD, and DiD-loaded NPs, RNPs, and PRNPs in AIA rats with a unilateral inflamed joint at $24 \mathrm{~h}$ post injection. $\mathbf{c}$ Ex vivo DiD fluorescence images in the inflamed joints and un-inflamed joints from AIA rats with a unilateral inflamed joint at $24 \mathrm{~h}$ post injection with free DiD, and DiD-labeled NPs, RNPs, or PRNPs. $\mathbf{d}$ The statistical graphs of the fluorescence intensity of inflamed joints based on the semi-quantitative analysis of the ex vivo fluorescence images after i.v. administration of free DiD or DiD-labeled nanoparticles. Data represent mean \pm SD ( $n=6$ inflamed joints from 3 independent animals). Statistical significance was determined by two-sided Student's $t$ test. e The statistical graphs of the fluorescence intensity of major organs from AIA rats with advanced arthritis after i.v. administration of free DiD or DiD-labeled nanoparticles. Data represent mean \pm SD ( $n=3$ independent animals). Statistical significance was determined by a two-sided Student's $t$ test. $\mathbf{f}$ The statistical graphs of the fluorescence intensity of inflamed joints and un-inflamed joints from AIA rats with a unilateral inflamed joint after i.v. administration of free DiD or DiD-labeled nanoparticles. Data represent mean \pm SD $(n=3$ independent animals). Statistical significance was determined by a two-sided Student's $t$ test. DiD 1,1'-dioctadecyl-3,3,3',3'-tetramethyl indodicarbocyanine, 4-chlorobenzenesulfonate salt, DiD-NPs DiD labeled poly (D, Llactide-co-glycolide) (PLGA) nanoparticles, DiD-RNPs DiD-labeled RGD peptide-modified PLGA nanoparticles, DiD-PRNPs DiD-labeled matrix metalloproteinase 9 (MMP9)-cleavable polyethylene glycol (PEG)- and RGD peptide-modified PLGA nanoparticles.

of apoptosis in inflamed joints among all of the treatment groups. Furthermore, apoptotic cells induced by CEL-PRNPs were mainly inflammatory macrophages and OCs (Supplementary Fig. 11).

To determine the abundance of OCs and synovial macrophages in inflamed joints, ankle joints, and blood were collected for TRAP staining, immunohistochemical staining, and enzyme- linked immunosorbent assays (ELISA) 2 days after the last treatment. OCs located in the areas of bone erosion are stained red when using TRAP staining assay. TRAP staining results showed that CEL-PRNPs significantly reduced the number of OCs in ankle joints and resulted in negligible bone erosion (Fig. 7b). ELISA results revealed that the serum TRAP in the 


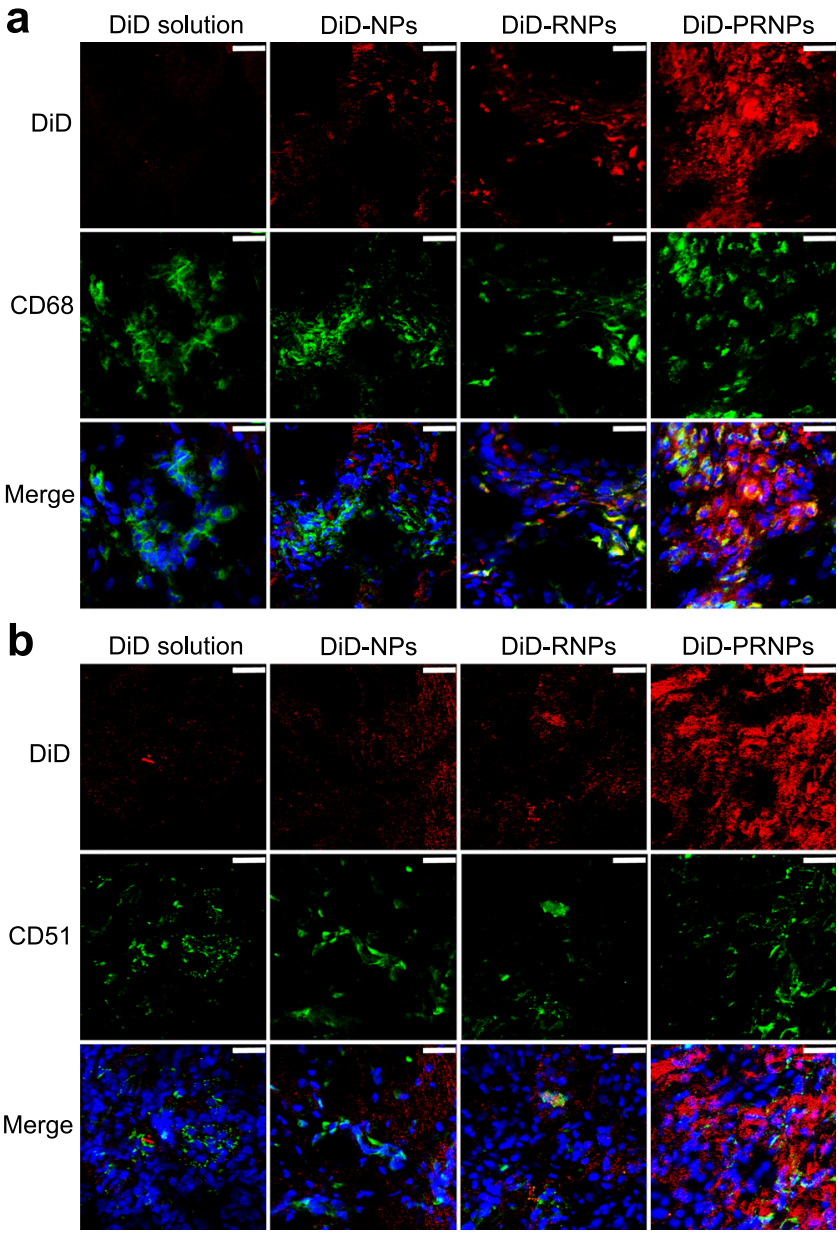

Fig. 6 PRNPs are selectively distributed in OCs and inflammatory macrophages in arthritic joints of rats with advanced arthritis. Confocal images showing the distribution of different DiD formulations in synovial macrophages (a) and OCs (b) in inflamed joints. Macrophages and OCs were determined by immunofluorescence analysis of CD68 and CD51 (green fluorescence), respectively. (Scale bar $=25 \mu \mathrm{m})(n=3$ independent animals). DiD 1,1'-dioctadecyl-3,3,3',3'-tetramethyl indodicarbocyanine, 4chlorobenzenesulfonate salt, DiD-NPs DiD labeled poly ( $\mathrm{D}$, L-lactide-coglycolide) (PLGA) nanoparticles, DiD-RNPs DiD-labeled RGD peptidemodified PLGA nanoparticles, DiD-PRNPs DiD-labeled matrix metalloproteinase 9 (MMP9)-cleavable polyethylene glycol (PEG)- and RGD peptide-modified PLGA nanoparticles.

CEL-PRNPs group was comparable to that of the normal group (Supplementary Fig. 12). Immunohistochemical staining showed that, in comparison with free CEL, CEL-NPs, and CEL-RNPs treatment with CEL-PRNPs remarkably decreased the number of $\mathrm{CD}^{+}{ }^{+}$macrophages in the synovium (Fig. 7b). These results suggested that CEL-PRNPs efficiently reduced the abundance of both OCs and inflammatory macrophages in AIA rats with advanced arthritis.

The superabundant presence of OCs in RA severely disrupts the balance of bone function ${ }^{60,61}$. To confirm the possibility that CEL-PRNPs could restore bone function balance by decreasing OCs abundance, we determined the expression levels of RANKL and osteoprotegerin (OPG) in arthritic joints. Previous studies have demonstrated that the RANKL-OPG system is important for regulating the balance between bone resorption and formation $^{62,63}$ and increased RANKL expression and a higher RANKL/OPG ratio may contribute toward the inefficient bone erosion repair in $\mathrm{RA}^{64,65}$. We observed that the CEL-PRNPs group maintained a lower level of RANKL expression and a smaller RANKL/OPG ratio in the arthritic joints and blood than CEL, CEL-NPs, and CEL-RNPs groups, at a level comparable to that of the normal group (Fig. 7c; Supplementary Figs. 13 and 14), thereby demonstrating the recovery of bone function balance. In accordance with this, CEL-PRNPs promoted bone damage repair, as indicated by the significant accumulation of osteocalcin (OCN)-positive osteoblasts and the increased expression of alkaline phosphatase (ALP) in the arthritic joints (Fig. 7d; Supplementary Fig. 15).

Macrophages are the main producers of inflammatory cytokines including TNF and IL-1 $\beta$ in $\mathrm{RA}^{66,67}$. We found that the CEL-PRNPs group had the lowest expression levels of TNF and IL-1 $\beta$ in blood among all of the treatment groups (Fig. 7c). The immunohistochemical assays similarly revealed that CELPRNPs effectively reduced the secretion of TNF and IL-1 $\beta$ in ankle joints (Fig. 7d; Supplementary Fig. 15). Thus, CEL-PRNPs likely mediated the reduction of inflammatory macrophage abundance in arthritic joints. The above results demonstrated the marked ability of CEL-PRNPs in restoring bone function balance and reducing inflammatory cytokines secretion, through the reduction of the number of OCs and inflammatory macrophages in arthritic joints.

CEL-PRNPs alleviate joint inflammation and bone erosion in rats. Finally, the therapeutic efficacy of CEL-PRNPs treatment was evaluated in AIA rats with advanced arthritis. The AIA rat developed severe swelling in the ankles and paws after 17 days of arthritis induction. Saline or various CEL-loaded PLGA nanoparticles were intravenously injected into rats (dose of $1 \mathrm{mg} / \mathrm{kg}$ for CEL) (Fig. 8a). Anti-TNF, as one of the benchmarks in the treatment of RA, was also employed to treat AIA rats with advanced arthritis in this study. Free CEL and anti-TNF showed relatively low efficacy in decreasing the paw thickness and ankle diameters of AIA rats with advanced arthritis. In contrast, CELPRNPs showed higher efficacy in reducing swelling in ankle joints and paws compared with CEL-NPs and CEL-RNPs, yielding an ankle diameter and paw thickness closer to that of the normal group at the study endpoint (Fig. 8b, c). To further illustrate that CEL-PRNPs could control inflammation and reduce cartilage destructions, the ankle joints of rats were sectioned for histological analysis at the study endpoint. H\&E-stained sections from the saline group showed severe synovial hyperplasia, along with bone and cartilage destruction. The free CEL group displayed a limited effect in reducing these symptoms, while CEL-NPs and CEL-RNPs reduced synovial inflammation and decreased the loss of cartilage to some extent compared with the saline group (Fig. 8d). However, safranin-O and toluidine blue staining revealed that the GAG levels in CEL-NPs and CEL-RNPs were lower than those in the control AIA rats (AIA rats following 17 days of arthritis induction) (Supplementary Fig. 1), suggesting that the cartilage damage was inefficiently reversed and remained progressive. In contrast, $\mathrm{H} \& \mathrm{E}$ results showed mild synovial hyperplasia in the CEL-PRNPs group. Additionally, the CELPRNPs group had larger positive areas for safranin-O and toluidine blue staining, which were closer to those of the normal group (Fig. 8d; Supplementary Fig. 16). These results demonstrated that CEL-PRNPs effectively alleviated synovial inflammation and reduced cartilage destructions in AIA rats with advanced arthritis.

We proved that AIA rats following 17 days of disease induction had obvious bone erosion and significant loss of BMD (Fig. 1). Using the micro-CT analysis, the inflamed ankle joints on day 29 after treatment were shown to exhibit rough bone surfaces and serious bone erosion in the saline group, with a significant 

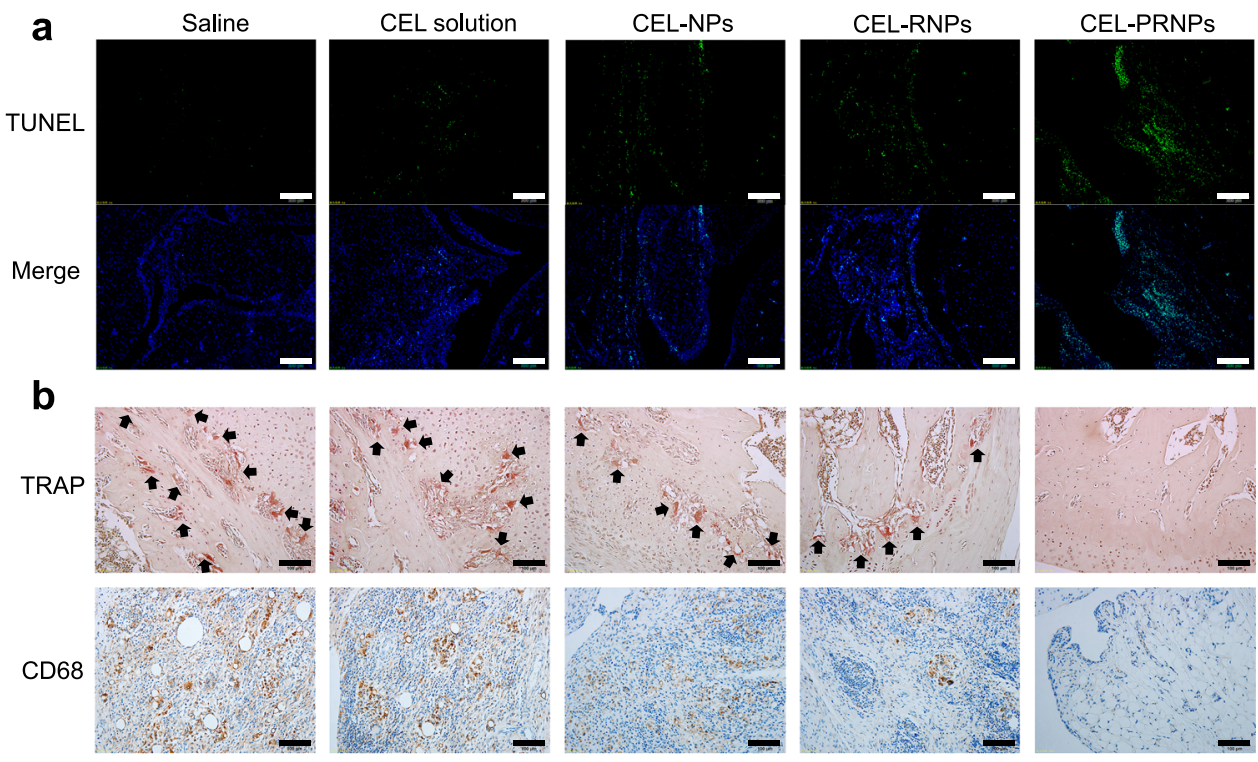

C
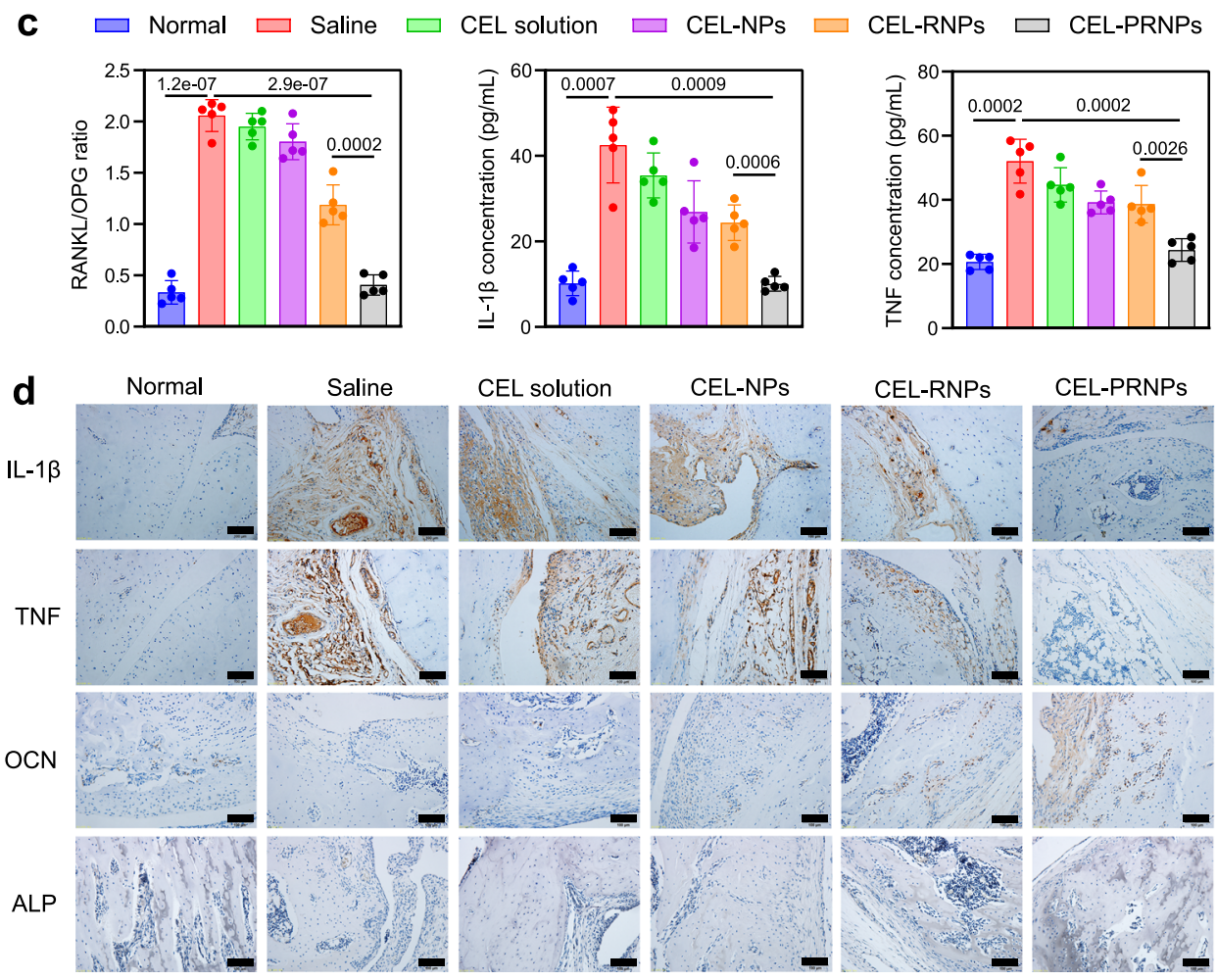

Fig. 7 CEL-PRNPs reduce the number of OCs and inflammatory macrophages in joints of rats with advanced arthritis. a TUNEL immunofluorescence staining in ankle joints from AIA rats receiving the indicated treatment (Scale bar $=200 \mu \mathrm{m})(n=5$ independent animals). b Immunohistochemical analyses of the TRAP-stained OCs and CD68-stained synovial macrophages in the joint tissues from rats receiving the indicated treatment (Scale bar = $100 \mu \mathrm{m})(n=5$ independent animals). c RANKL/OPG ratio in arthritic joints, IL-1 $\beta$ secretion in blood, and TNF secretion in blood from rats receiving the indicated treatment. Data represent mean \pm SD ( $n=5$ independent animals). Statistical significance was determined by a two-sided Student's $t$ test. d Detection of IL-1 $1 \beta, T N F, O C N$, and ALP expression levels in arthritic joints in different groups. Arthritic joints in different groups were stained with IL-1 $\beta$, TNF, and OCN antibodies, respectively. ALP was stained light-dark in arthritic joints from different groups (Scale bar $=100 \mu \mathrm{m})(n=5$ independent animals). CEL celastrol CEL-NPs CEL-loaded poly (D, L-lactide-co-glycolide) (PLGA) nanoparticles, CEL-RNPs CEL-loaded RGD peptide-modified PLGA nanoparticles, CEL-PRNPs CEL-loaded matrix metalloproteinase 9 (MMP9)-cleavable polyethylene glycol (PEG)- and RGD peptide-modified PLGA nanoparticles, TUNEL TdT-mediated dUTP nick end labeling, TRAP tartrate-resistant acid phosphatase, RANKL receptor of activator of NF-kB ligand, OPG osteoprotegerin, IL-1 $\beta$ interleukin-1 $\beta$, TNF tumor necrosis factor, OCN osteocalcin, ALP alkaline phosphatase.

reduction in $\mathrm{BMD}$ and an increase in $\mathrm{BS} / \mathrm{BV}$ compared with the normal group. Free CEL showed moderate efficacy in reducing bone erosion. Treatment with anti-TNF, CEL-NPs, and CELRNPs increased the BMD compared with free CEL but failed to repair the bone damage. Remarkably, the CEL-PRNPs group showed smooth bone surfaces and had a high BMD, closer to those of the normal group (Fig. 9a-d), demonstrating that CELPRNPs efficiently reversed bone erosion. The trabecular 
a

\begin{tabular}{ccccc} 
Day 0 & Day 23 & Day 26 & Day 29 \\
Arthritis induction 20 & $\begin{array}{c}\text { Advanced arthritis } \\
\text { established }\end{array}$ & $\begin{array}{c}\text { Rat } \\
\text { sacrificed }\end{array}$ \\
\hline
\end{tabular}

b

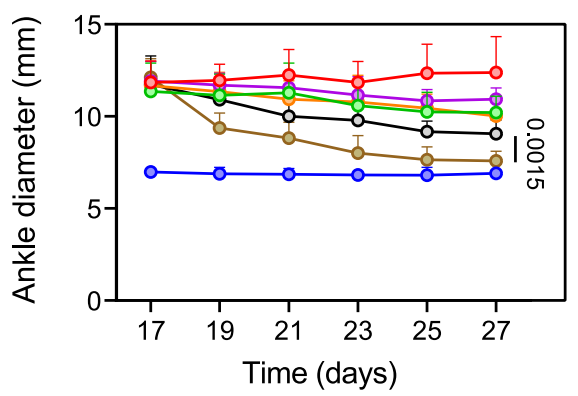

C

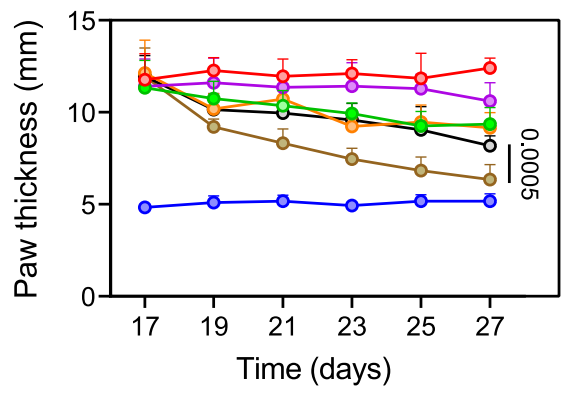

-0 Normal

-o- Saline

$\rightarrow$ Anti-TNF

-o- CEL solution

- - CEL-NPs

-o- CEL-RNPS

-o- CEL-PRNPS

d
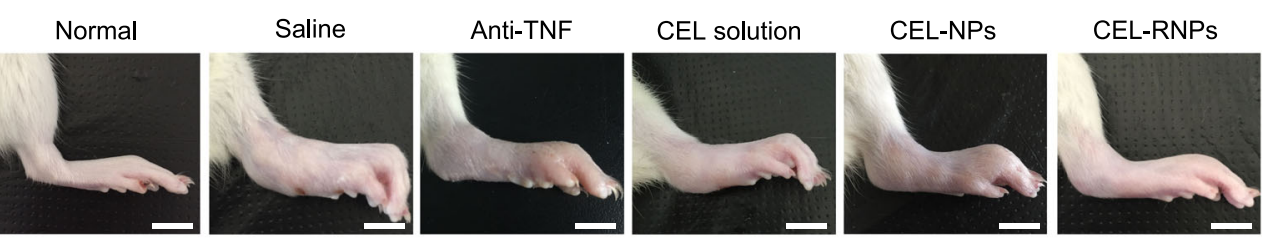

CEL-PRNPs
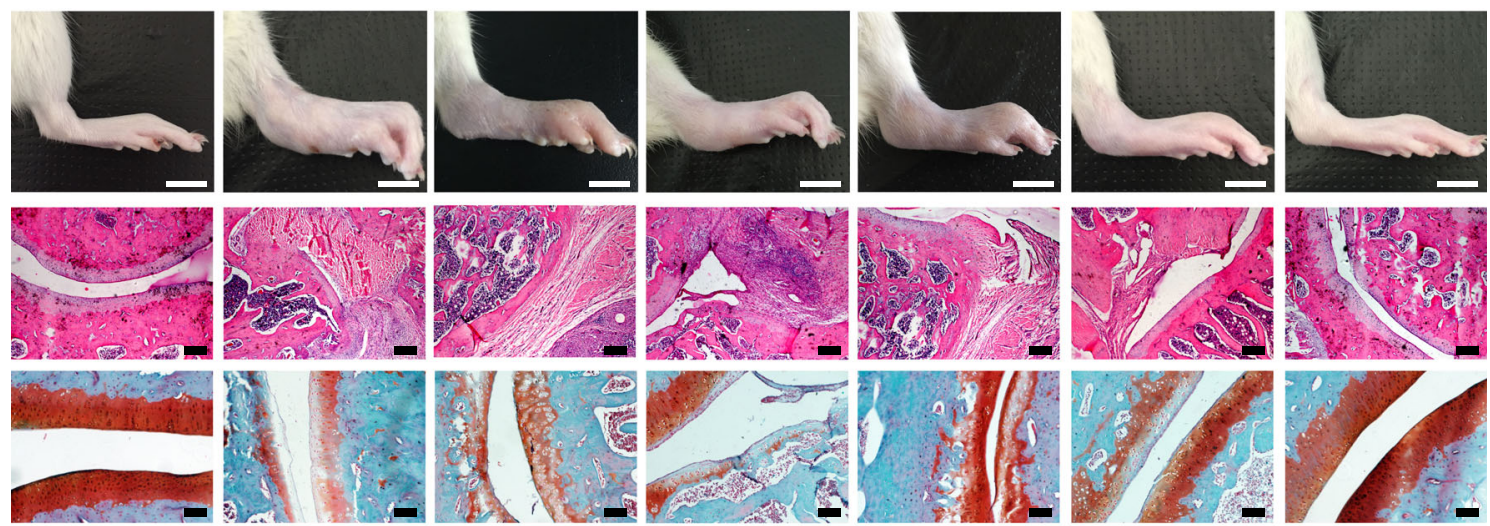

Safranin O
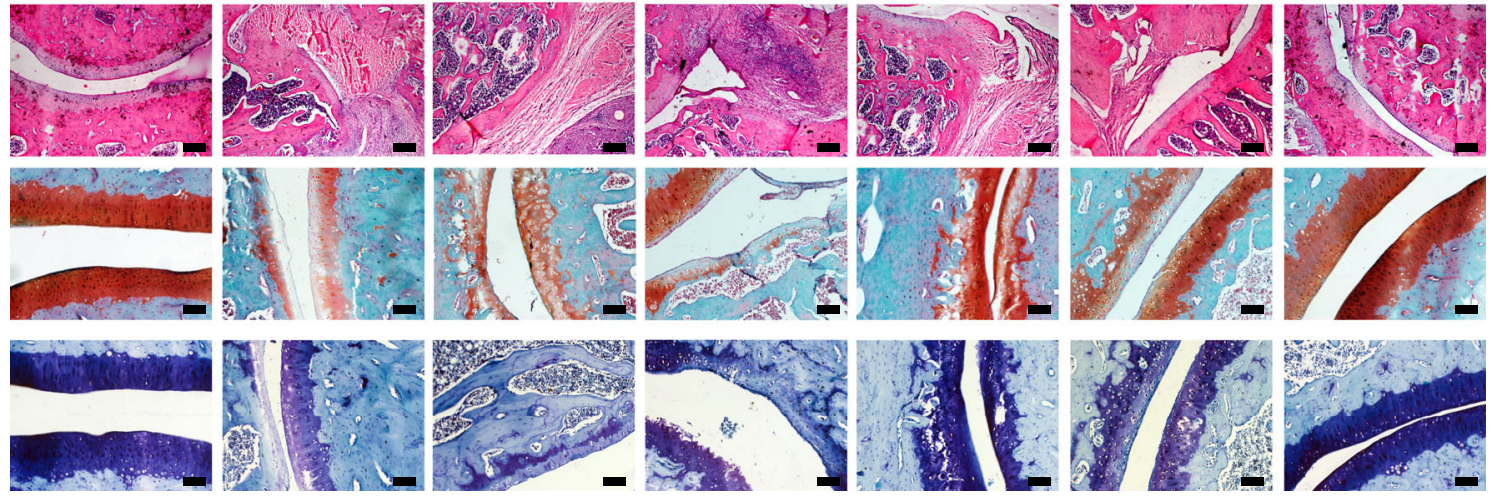

Fig. 8 Therapeutic efficacy of CEL-PRNPs in rats with advanced arthritis. a The schematic illustration of CEL-PRNPs treatment. b, c Ankle diameter (b) and paw thickness (c) of AIA rats were recorded every other day during the treatment period. Data represent mean \pm SD ( $n=7$ independent animals). Statistical significance was determined by a two-sided Student's $t$ test. d Representative photographs of hindlimbs at the endpoint of the experiment from different treatment groups (Scale bar $=10 \mathrm{~mm}$ ); histopathology evaluation of ankle joints was identified using $\mathrm{H} \& \mathrm{E}(\mathrm{scale}$ bar $=200 \mu \mathrm{m}$ ), safranin-O and toluidine blue staining (scale bar $=100 \mu \mathrm{m})(n=5$ independent animals). i.v. intravenous, anti-TNF anti-TNF (tumor necrosis factor) antibody, CEL celastrol, CEL-NPs CEL-loaded poly (D, L-lactide-co-glycolide) (PLGA) nanoparticles, CEL-RNPs CEL-loaded RGD peptide-modified PLGA nanoparticles, CEL-PRNPs CEL-loaded matrix metalloproteinase 9 (MMP9)-cleavable polyethylene glycol (PEG)- and RGD peptide-modified PLGA nanoparticles, H\&E hematoxylin-eosin.

parameters also confirmed that CEL-PRNPs treatment was the most efficient in increasing the trabecular number (Tb.N) and trabecular bone thickness (Tb.Th) while decreasing trabecular separation (Tb.Sp) among all of the treatment groups (Fig. 9e-g). Thus, these findings proved that CEL-PRNPs could effectively terminate the progression of bone damages and simultaneously repair bone erosion in a rat model of advanced RA.

\section{Discussion}

Apoptosis is the process of natural programmed cell death used to maintain organism homeostasis ${ }^{68}$. For example, macrophages are the first line of defense against pathogens ${ }^{69,70}$. They are regulated by apoptosis to maintain immune homeostasis and protect the host against damage from excessive inflammation ${ }^{21,22}$. OCs are terminally differentiated cells and known for their unique function in promoting bone resorption and the formation of resorption lacunae ${ }^{14-16}$. The natural apoptosis of OCs helps to balance body bone-remodeling homeostasis to prevent OCsmediated bone damage 23,54 . However, macrophages and OCs are abnormally accumulated in RA joints ${ }^{10,11,66}$. Furthermore, macrophages and OCs in arthritic joints are reported to exhibit the decreased apoptotic rates compared with those from healthy controls $^{21,25}$. Therefore, we proposed that enhancing the apoptosis of macrophages and OCs would be a promising treatment strategy for restoring immune homeostasis and bone function balance in arthritic joints.

Our previous study found that CEL could trigger the apoptosis of mesangial cells in glomerulonephritis ${ }^{38}$. In the present study, we demonstrated the CEL-induced apoptosis of pathogenic macrophages and OCs (Figs. $3 \mathrm{~b}$ and $4 \mathrm{e}, \mathrm{f}$ ). However, CEL treatment shows severe toxicity in normal organs due to its offtargeting activity ${ }^{38,71}$. Indeed, significant CEL toxicity in the 
a

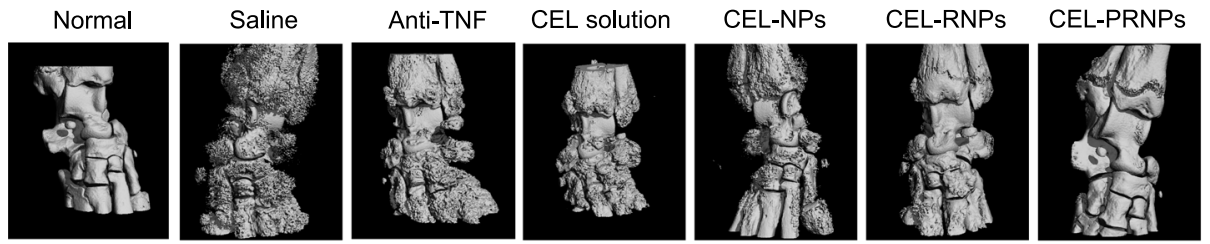

b

Normal

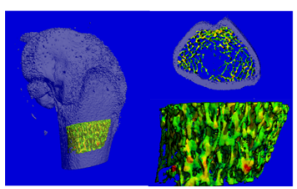

Saline

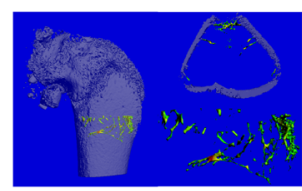

CEL-RNPs

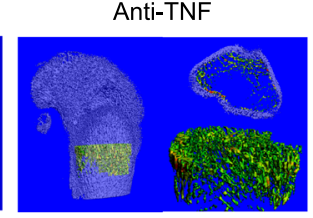

CEL solution

CEL-NPs

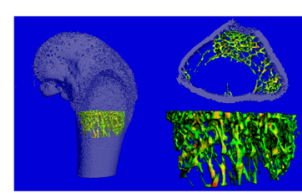

CEL-PRNPs
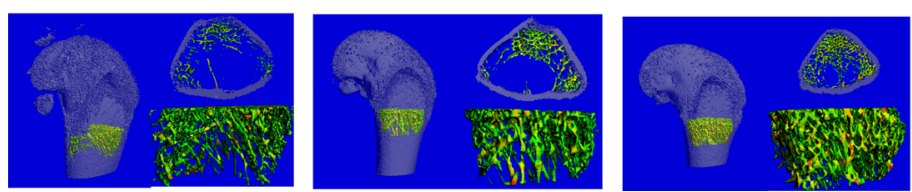

C

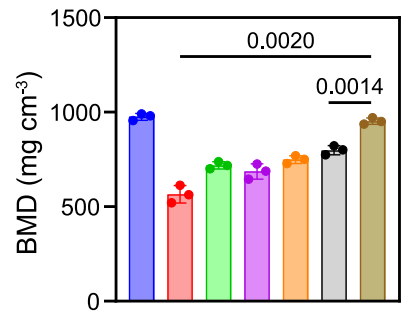

d

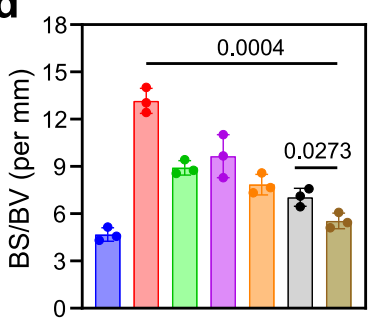

e

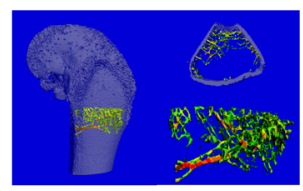

f

g
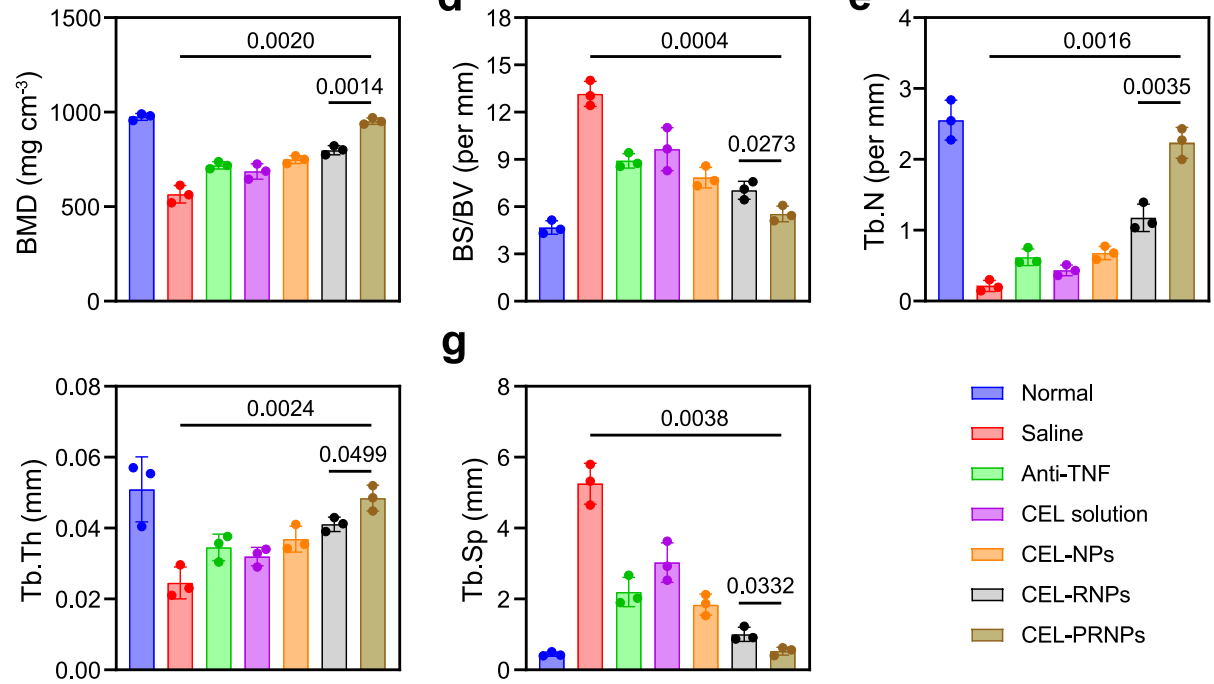

$$
\begin{aligned}
& \square \text { Normal } \\
& \square \text { Saline } \\
& \square \text { Anti-TNF } \\
& \square \text { CEL solution } \\
& \square \text { CEL-NPs } \\
& \square \text { CEL-RNPs } \\
& \square \text { CEL-PRNPs }
\end{aligned}
$$

Fig. 9 CEL-PRNPs reverse bone erosion in rats with advanced arthritis. a Representative micro-CT images of the ankle joints at the endpoint of the experiment from different treatment groups in therapeutic efficacy study ( $n=3$ independent animals). $\mathbf{b}$ Representative micro-CT images of the trabecular in bone and the reconstructed trabecular structure $(n=3$ independent animals). c, d Quantitative micro-CT analysis of BMD (c) and BS/BV (d) of the ankle joints at the endpoint of the experiment. Data represent mean \pm SD ( $n=3$ independent animals). Statistical significance was determined by a twosided Student's $t$ test. e-g Quantitative micro-CT analysis of trabecular number (Tb.N) (e), trabecular bone thickness (Tb.Th) (f), and decreasing trabecular separation (Tb.Sp) (g). Data represent mean \pm SD ( $n=3$ independent animals). Statistical significance was determined by a two-sided Student's $t$ test. Anti-TNF anti-TNF (tumor necrosis factor) antibody, CEL celastrol, CEL-NPs CEL-loaded poly (D, L-lactide-co-glycolide) (PLGA) nanoparticles, CELRNPs CEL-loaded RGD peptide-modified PLGA nanoparticles, CEL-PRNPs CEL-loaded matrix metalloproteinase 9 (MMP9)-cleavable polyethylene glycol (PEG)- and RGD peptide-modified PLGA nanoparticles.

heart, liver, and brain was also observed in our study (Supplementary Figs. 17 and 18). Thus, it is necessary to specifically induce the apoptosis of inflammatory macrophages and OCs in arthritic joints to treat RA. To realize cell-specific drug delivery in the treatment of RA, the drug delivery system should be able to target both the inflammatory sites and the relevant cells within these sites. The RGD peptide is a well-known ligand of the integrins that are highly expressed on the surfaces of pathogenic macrophages and OCs in RA synovium ${ }^{19,20}$. Thus, we observed significant uptake of RGD-modified NPs (RNPs) by pathogenic macrophages (LPS-activated murine macrophages and human synovial macrophages) and OCs (Figs. 3a and 4a). However, integrins are overexpressed in many organs such as the liver ${ }^{59}$, and this led to the enhanced distribution of RNPs in the liver and higher CEL toxicity in this organ (Fig. 5a; Supplementary Figs. 17 and 18). Furthermore, integrins are also highly expressed on the neovascularization endothelial cells within the inflammatory sites of RA, which could lead to the selective distribution of RGD modified nanoparticles in endothelial cells ${ }^{72,73}$. This selective uptake by endothelial cells would hinder these nanoparticles from reaching the inflammatory microenvironment in RA. Our results also revealed that RNPs and PRNPs (in the presence of MMP9) exhibited high uptake by TNF-activated HUVECs, which mimic neovascularization endothelial cells. However, as a result of their MMP9-responsive PEG chain modification, PRNPs showed decreased distribution in activated HUVECs (Supplementary Fig. 19). Therefore, PRNPs with their inflammatory microenvironment-responsive properties offer not only good 
in vivo safety but also enable the selective delivery of CEL-RNPs to the macrophages and OCs within the inflammatory microenvironment of arthritis.

Macrophages in the RA synovium produced cytokines, such as TNF and IL-1, to promote the progression of inflammation ${ }^{66,67}$. Thus, anti-cytokines are often applied in the clinical treatment of RA. In our rat RA model, we also found that anti-TNF treatment could significantly suppress the inflammation in early arthritis (Supplementary Fig. 20). However, the anti-TNF treatment showed reduced efficacy and limited bone protection in advanced arthritis (Figs. 8 and 9). What's more, anti-TNF and anti-IL-1 therapies are clinically effective in only $40 \%$ of patients, and the disease recurs when treatment is stopped ${ }^{21,30,32}$. This might be due to synovial macrophages producing other inflammatory mediators besides TNF and IL-1, such as IL- 6 and IL-15 $5^{66,74}$. In addition, synovial macrophages have a prolonged life span due to their insufficient apoptosis ${ }^{21,75}$. In contrast with anti-TNF treatment, CEL-loaded nanoparticles can target the synovial macrophages as a whole to induce their apoptosis. Furthermore, our CEL-PRNPs could selectively deliver CEL to inflammatory macrophages and OCs in arthritic joints to effectively induce the apoptosis of both cell types, thus significantly suppressing inflammation and terminating bone erosion (Figs. 8 and 9). Of note, the effective OCs depletion induced by CEL-PRNPs efficiently restored the balance of bone function, as indicated by the reduced RANKL expression and decreased RANKL/OPG ratio (Fig. 7c; Supplementary Figs. 13 and 14). The recovery of bone function balance induced by CEL-PRNPs promoted bone erosion repair by increasing ALP expression and the accumulation of OCN-positive osteoblasts (Fig. 7d).

Secondary osteoporosis occurs widely among RA patients because the increased number of OCs in RA severely disrupts the OC-osteoblast axis and enhances the bone resorption function of OCs ${ }^{12,76}$. Furthermore, glucocorticoids, one of the most common anti-inflammatory agents, are frequently applied in the treatment of patients with RA. Unfortunately, one severe side effect of glucocorticoids is osteoporosis induced by the prevention of calcium absorption ${ }^{29}$. Interestingly, the dual-targeting scaffold CEL-PRNPs developed in this study could effectively increase the bone density, trabecular number, and bone thickness, and decrease the trabecular separation, thereby demonstrating the efficient control of osteoporosis in advanced arthritis (Fig. 9). Such phenomena were mainly attributed to the enhanced OCs apoptosis induced by CEL-PRNPs effectively restoring the bone function balance. Therefore, our findings might suggest a new direction in nanomedicine in the osteoporosis control of RA patients.

PLGA, PEG, and RGD have been approved in clinic application and the substrate peptide of MMP9 also consists of essential amino acids for humans. CEL-PRNPs treatment showed good efficacy and led to negligible off-target apoptosis in rats. But further clinical translation, long-term toxicity of using PRNPs should be thoroughly evaluated in the future. In addition, knockout mice ( $\alpha \mathrm{v} \beta 3$ integrin KO mice and $\mathrm{MMP}^{-1-}$ mice) could be adopted to evaluate the efficacy of CEL-PRNPs. The in vivo studies in knockout mice will be advantageous to the clinical translation of this developed drug delivery platform.

In summary, we developed inflammatory macrophages and OCs dual-targeted strategy based on MMP9-responsive CELPRNPs for advanced inflammatory arthritis treatment. CELPRNPs were shown to efficiently target both inflammatory macrophages and OCs after responding to MMP9 in the inflammatory microenvironment of arthritis. Targeting of inflammatory macrophages promoted their apoptosis, thereby reducing inflammation in arthritic joints. Targeting of OCs promoted OC apoptosis and inhibited their osteoclastic function, consequently restoring the balance of bone function. Accordingly, CEL-PRNPs efficiently controlled joint inflammation, reversed bone erosion, and prevented secondary osteoporosis. Taken together, CEL-PRNPs realized the targeting of both synovial inflammation and bone erosion in advanced arthritis. This strategy for the selective apoptosis of inflammatory macrophages and OCs in arthritic joints shows great promise in inflammatory remission, bone erosion repair, and secondary osteoporosis prevention in advanced inflammatory arthritis.

\section{Methods}

Materials. CEL (Catalog \# A0106) was obtained from Chengdu Must Biotechnology (Chengdu, China). Poly (ethylene glycol)-poly (D, L-lactide-co-glycolide 50/50) $\left(\mathrm{PEG}_{2000}-\mathrm{PLGA}_{20000}\right)$ and maleimide- $\mathrm{PEG}_{2000}-\mathrm{PLGA}_{20000}\left(\mathrm{Mal}_{-} \mathrm{PEG}_{2000^{-}}\right.$ $\mathrm{PLGA}_{2000}$ ) were obtained from the University of Electronic Science and Technology of China (Chengdu, China). Cys-RGD peptide and $\mathrm{mPEG}_{2000}-\mathrm{MMP} 9$ cleavable Cys-peptide ( $\mathrm{mPEG}_{2000}$-GPLGLAGQC) were custom-synthesized by GL Biochem (Shanghai) Ltd. (Shanghai, China). RANKL (Catalog \# 315-11) and MCSF (Catalog \# 315-02) were obtained from PeproTech (Rocky Hill, USA). 1,1'dioctadecyl-3,3,3',3'-tetramethyl indodicarbocyanine, 4-chlorobenzenesulfonate salt (DiD) (Catalog \# M9379) was obtained from ChemBridge (San Diego, USA) LPS (Catalog \# L4391) and human active MMP9 protein (Catalog \# PF024) were purchased from Sigma Aldrich Co., LLC. (St. Louis, USA). Annexin V-FITC Apoptosis/Propidium Iodide Detection Kit (Catalog \# G003-1-3) and TRAP Stain Kit (Catalog \# D023-1-1) were from Nanjing Keygen Biotech. Co., Ltd. (Nanjing, China). JC-1 molecular probe (Catalog \# C2005) was acquired from Beyotime Institute of Biotechnology (Haimen, China). In Situ Cell Death Detection Kit (Catalog \# 11684795910) for TUNEL assay was from Roche (Basel, Switzerland). ELISA kits to assay the levels of rat RANKL (Catalog \# SBJ-R0631) and OPG (Catalog \# SBJ-R0631) were obtained from Nanjing Senbeijia Biological Technology Co., Ltd. (Nanjing, China). ELISA kits to assay the levels of rat TNF (Catalog \# KRC3011) and IL-1 $\beta$ (Catalog \# BMS630) were purchased from Invitrogen (Shanghai, China).

Patient samples. Peripheral blood samples and synovial tissues were obtained from three female patients with late-stage RA (according to the American College of Rheumatology criteria) undergoing joint replacement surgery. All samples were collected from Xiangya Hospital of Central South University. Informed consent was obtained from all patients, and ethical approval was obtained from the Ethics Committee of the Xiangya Hospital of Central South University (approval No. 2019010305)

Animals. Healthy male Wistar rats $(200 \pm 20 \mathrm{~g}, 5$ weeks old $)$ and male C57BL/J mice ( $20 \pm 2$ g, 6 weeks old) were obtained from Chengdu Dashuo Experimental Animal Co., Ltd. (Chengdu, China). Animals were housed in specific pathogen-free conditions at a standard temperature of $22 \pm 2{ }^{\circ} \mathrm{C}$ and a relative humidity of $55 \%$ (45-70\%) in a 12:12-h light-dark cycle. All animal studies were conducted according to the requirements of the national act regarding the use of experimental animals (China) and complied with the guidelines evaluated and approved by the Animal Ethics Committee of Sichuan University.

Cells. The in vitro OC differentiation was carried out as previously described ${ }^{77}$. In brief, bone marrow cells were isolated from the tibiae of C57BL/J mice and these cells were cultured with $30 \mathrm{ng} / \mathrm{mL}$ of M-CSF for 2 days and used as BMMs. To generate OCs, the BMMs were cultured in the presence of $100 \mathrm{ng} / \mathrm{mL}$ of RANKL and $30 \mathrm{ng} / \mathrm{mL}$ of M-CSF for 4 days. To obtain LPS-activated macrophages, BMMs were treated with $10 \mathrm{ng} / \mathrm{mL}$ of LPS for $48 \mathrm{~h}$. All cells were cultured in RPMI-1640 medium containing $10 \% \mathrm{FBS}$ and $100 \mathrm{U} / \mathrm{mL}$ of penicillin-streptomycin under $5 \%$ $\mathrm{CO}_{2}$ at $37^{\circ} \mathrm{C}$

Synovial tissue specimens were gained from patients with late-stage RA undergoing joint replacement surgery. The gained tissue specimens were washed, cut into small pieces and digested with collagenase. Tissue debris was removed by forcing the sample through a $70-\mu \mathrm{m}$ cell strainer, thereby producing a cell suspension. Magnetic-activated cell sorting method ${ }^{78,79}$ was adopted to isolate the macrophages from synovium-derived cells. Synovial macrophages were isolated to a high percentage of purity $(>95 \%)$ with the use of MACS CD14 MicroBeads (Miltenyi Biotech, Germany). Primary synovial macrophages were cultured in Dulbecco's Modified Eagle's Medium (DMEM) containing 10\% FBS and $100 \mathrm{U} / \mathrm{mL}$ of penicillin-streptomycin under $5 \% \mathrm{CO}_{2}$ at $37^{\circ} \mathrm{C}$.

Blood samples were obtained from patients diagnosed with late-stage RA. Peripheral blood mononuclear cells (PBMCs) were isolated using Ficoll-Paque (Miltenyi Biotech) density gradient centrifugation according to the manufacturer's instructions. PBMCs were cultured in the presence of $100 \mathrm{ng} / \mathrm{mL}$ of RANKL and $30 \mathrm{ng} / \mathrm{mL}$ of M-CSF for 4 days to generate OCs. RA patients-derived OCs were cultured in RPMI-1640 medium containing $10 \% \mathrm{FBS}$ and $100 \mathrm{U} / \mathrm{mL}$ of penicillin-streptomycin under $5 \% \mathrm{CO}_{2}$ at $37^{\circ} \mathrm{C}$. 
Human umbilical vein endothelial cells (HUVECs) were purchased from the Chinese Academy of Sciences Cell Bank for Type Culture Collection (Shanghai, China). HUVECs were cultured in RPMI-1640 medium containing 10\% FBS and $100 \mathrm{U} / \mathrm{mL}$ of penicillin-streptomycin under $5 \% \mathrm{CO}_{2}$ at $37^{\circ} \mathrm{C}$. To activate HUVECs, cells were treated with cells with $50 \mathrm{ng} / \mathrm{mL}$ of TNF for $24 \mathrm{~h}$.

Synthesis and characterization of RGD-PEG $\mathbf{2 0 0 0}_{\mathbf{2}} \mathbf{P L G A}_{\mathbf{2 0 0 0 0}}$. RGD-PEG $\mathrm{P}_{2000^{-}}$ $\mathrm{PLGA}_{20000}$ was synthesized via the maleimide-thiol coupling reaction. Briefly, Mal$\mathrm{PEG}_{2000}-\mathrm{PLGA}_{20000}$ and Cys-RGD (molar ratio = 1:2) were reacted in a solvent mixture comprising chloroform $/ \mathrm{MeOH}(\mathrm{v} / \mathrm{v}=2: 1)$ with gentle stirring at room temperature for $12 \mathrm{~h}$. The solvent was evaporated in a vacuum and the residue was re-dissolved with chloroform. The insoluble material (unreacted Cys-RGD) was filtered out, and the filtrate was evaporated in a vacuum to obtain RGD- $\mathrm{PEG}_{2000^{-}}$ $\mathrm{PLGA}_{20000}$. The successful synthesis of RGD-PEG $2000-\mathrm{PLGA}_{20000}$ was confirmed by ${ }^{1} \mathrm{H}-\mathrm{NMR}$.

Preparation and characterization of CEL-NPs, CEL-RNPs, and CEL-PRNPs. CEL-NPs were prepared using an emulsion/solvent evaporation method. Briefly, $\mathrm{PEG}_{2000}-\mathrm{PLGA}_{20000}$ and CEL were dissolved in chloroform to form the oil phase. The resultant oil phase was then added into an aqueous phase. The mixture was emulsified by sonication with a probe sonicator (Ningbo Xinzhi Biotechnology Co. Ltd.; Ningbo, China). CEL-NPs were then prepared after the chloroform was evaporated at low pressure. CEL-RNPs were prepared as indicated above using $\mathrm{PEG}_{2000}-\mathrm{PLGA}_{20000}, \mathrm{MAL}-\mathrm{PEG}_{2000}-\mathrm{PLGA}_{20000}$, and RGD-PEG ${ }_{2000}-\mathrm{PLGA}_{20000}$, and CEL was dissolved in chloroform to form the oil phase. To obtain CEL-PRNPs, $\mathrm{mPEG}_{2000}$-GPLGLAGQC was conjugated with CEL-RNPs in PBS with $\mathrm{pH} 7.4$ at room temperature for $4 \mathrm{~h}^{52}$. Unconjugated $\mathrm{mPEG}_{2000}$-GPLGLAGQC was removed by elution through a Sephadex G-75 column.

The particle sizes and zeta potentials of CEL-NPs, CEL-RNPs, and CEL-PRNPs were determined by dynamic light scattering (DLS) using a Zetasizer Nano ZS90 instrument (Malvern Panalytical, Malvern, UK). The encapsulation efficiency (EE \%) was determined by ultrafiltration method. The morphology of CEL-NPs, CELRNPs, and CEL-PRNPs was determined by TEM (H-600, Hitachi, Japan).

To study the serum stability, the prepared CEL-NPs, CEL-RNPs, and CELPRNPs were stored in $10 \% \mathrm{FBS}$ at $37^{\circ} \mathrm{C}$ for $24 \mathrm{~h}$. Changes in particle size were determined by DLS.

Cellular uptake study. BMMs, HUVECs, activated HUVECs, OCs, and macrophages derived from mice and patients were seeded in 12-well plates and were treated with C6-NPs, C6-RNPs, and C6-PRNPs (with or without $5 \mu \mathrm{g} / \mathrm{mL}$ of MMP9) in the serum-free medium. After a $1 \mathrm{~h}$ incubation, cells were collected, centrifuged, and then suspended in phosphate-buffered saline (PBS). The fluorescence intensity of C6 was measured by a flow cytometer (BD FACSCelesta, USA).

For the qualitative analysis of cellular uptake, cells seeded in glass-bottomed dishes were treated as above. After incubation with C6-loaded NPs for $1 \mathrm{~h}$, cells were fixed and stained with DAPI in the dark. The fluorescence images were then obtained using a laser scanning confocal microscope (Olympus FluoView FV 1000 , USA).

Apoptosis study. OCs and macrophages were treated with CEL-NPs, CEL-RNPs, or CEL-PRNPs (the equivalent of $100 \mathrm{ng} / \mathrm{mL}$ of CEL and with or without $5 \mu \mathrm{g} / \mathrm{mL}$ of MMP9) for $24 \mathrm{~h}$. Cell apoptosis was determined using an Annexin V-FITC Apoptosis/Propidium Iodide Detection Kit according to the manufacturer's instructions and the flow cytometric images were analyzed with FlowJo V10. The mitochondrial membrane potentials of these apoptotic macrophages were measured by incubating the macrophages with $5 \mu \mathrm{g} / \mathrm{mL}$ of JC- 1 for $30 \mathrm{~min}$. The fluorescent images were obtained using a laser scanning confocal microscope.

TRAP assay. To generate OCs, BMMs were cultured in the presence of $100 \mathrm{ng} / \mathrm{mL}$ of RANKL and $30 \mathrm{ng} / \mathrm{mL}$ of M-CSF for 4 days. The control group comprised BMMs cultured with M-CSF only for 4 days. At the study endpoint, cells were stained by TRAP Stain Kit and observed with a light microscope (Axiovert 40CFL, Germany).

AIA model. The AIA model was developed according to a previous study but with slight modifications ${ }^{80}$. In brief, healthy male Wistar rats $(200 \pm 20 \mathrm{~g})$ were subcutaneously injected with Freund's adjuvant $(200 \mu \mathrm{L})$ containing $10 \mathrm{mg} / \mathrm{mL}$ of heat-killed mycobacteria (Chondrex, Washington DC, USA) into the base of their tails. Rats with 4 days of disease induction were stipulated as AIA rats with earlystage arthritis and an advanced arthritis rat model was successfully developed 17 days after disease induction. In addition, AIA rats with a unilateral inflamed joint were induced by the subcutaneous injection of Freund's adjuvant $(200 \mu \mathrm{L})$ containing $10 \mathrm{mg} / \mathrm{mL}$ of heat-killed mycobacteria into the paw of the right hind limb.

Biodistribution of PRNPs. Arthritic rats with advanced arthritis were established and intravenously injected with free DiD, DiD-NPs, DiD-RNPs, or DiD-PRNPs via the tail vein. The biodistribution of DiD in the ankle joints was analyzed 2, 8, 24, and $48 \mathrm{~h}$ after administration using in vivo imaging analysis of DiD fluorescence with a Caliper IVIS Lumina III In Vivo Imaging System (Perkin Elmer, USA). At the end of this experiment, rats were sacrificed, and their blood, organs (heart, liver spleen, lung, and kidney), and ankle joints were collected for ex vivo imaging of DiD fluorescence.

AIA rats with a unilateral inflamed joint were intravenously injected with free DiD, DiD-NPs, DiD-RNPs, or DiD-PRNPs via the tail vein. The biodistribution of $\mathrm{DiD}$ in the ankle joints was analyzed $24 \mathrm{~h}$ after administration using in vivo imaging analysis of $\mathrm{DiD}$ fluorescence with a Caliper IVIS Lumina III In Vivo Imaging System (Perkin Elmer, USA). At the end of this experiment, rats were sacrificed, their hind limbs and forelimbs were collected for ex vivo imaging of DiD fluorescence.

Immunofluorescence staining. Arthritic rats with advanced arthritis were intravenously injected with free DiD, DiD-NPs, DiD-RNPs, or DiD-PRNPs via the tail vein. Ankle joints were collected to prepare sections $24 \mathrm{~h}$ after administration. The prepared sections of $10 \mu \mathrm{m}$ thickness were stained with rat anti-CD68 (Abcam, Cat\#ab125212, 1/500 dilution) and anti-CD51 (Abcam, Cat\#ab179475, 1/500 dilution). DAPI was used for the nuclear stain. The fluorescent distributions in synovial joints were observed with a laser scanning confocal microscope (Leica TCS SP8 CARS, Germany).

TUNEL staining. Saline or various CEL-loaded PLGA nanoparticles were respectively intravenously injected into RA rats (dose of $1 \mathrm{mg} / \mathrm{kg}$ for CEL). Rats have received this treatment twice, and 2 days after the last treatment, the rats were sacrificed and their arthritic joints were collected. The arthritic joints were sectioned for TUNEL, anti-CD68 (Abcam, Cat\#ab125212, 1/500 dilution), and antiCD51 (Abcam, Cat\#ab179475, 1/500 dilution) co-staining, then observed by a laser scanning confocal microscope and analyzed with Image J1.52.

Cytokine assay. AIA rats with advanced arthritis were established as indicated above. Saline or various CEL-loaded PLGA nanoparticles were respectively intravenously injected into rats (dose of $1 \mathrm{mg} / \mathrm{kg}$ for CEL). The rats received this treatment four times and blood samples were collected at the study endpoint. The blood samples were centrifuged at $2295 \mathrm{~g}$ for $15 \mathrm{~min}$ at $4{ }^{\circ} \mathrm{C}$ to obtain the serum. TNF, IL-1 $\beta$, RANKL, and OPG in the serum were detected using ELISA kits.

Therapeutic efficacy study. AIA rats with early-stage or advanced arthritis were used in the therapeutic efficacy study. Saline or $1 \mathrm{mg} / \mathrm{kg}$ of CEL equivalents of CEL solution, CEL-NPs, CEL-RNPs, or CEL-PRNPs were intravenously injected into rats every 3 days. Anti-rat TNF $(1.5 \mathrm{mg} / \mathrm{kg})$ was used as the positive control and injected intraperitoneally into rats every other two days. Paw thickness and the dimensions of ankle joints were measured every other day during the treatment.

Histology and immunohistochemical study. Ankle joints collected before the treatment and 2 days after the last treatment were fixed in $4 \%$ paraformaldehyde. Fixed ankle joints were then decalcified by daily changes of a $15 \%(\mathrm{w} / \mathrm{v})$ tetrasodium ethylenediaminetetraacetic acid solution for 2 months. The decalcified joints were subsequently embedded in paraffin and then sectioned for $\mathrm{H} \& \mathrm{E}$, safranin O, toluidine blue, TRAP, CD68, MMP9, RANKL, OPG, OCN, ALP, TNF, and IL-1 $\beta$ staining. Ankle joints from both the early- and late-stage of AIA rats were also obtained for H\&E, safranin O, toluidine blue, TRAP, CD68, and MMP9 staining. These sections were observed by a light microscope and analyzed with Image J1.52. anti-CD68: Abcam, Cat\#ab125212, 1/500 dilution; anti-MMP9: Abcam, Cat\#ab76003, 1/1000 dilution; anti-RANKL: Abcam, Cat\#ab239607, 1/100 dilution; anti-OPG: Abcam, Cat\#ab203061, 1/200 dilution; anti-OCN: Abcam, Cat\#ab13420, 1/200 dilution; anti-ALP: Abcam, Cat\#ab224335, 1/200 dilution; anti-TNF: Abcam, Cat\#ab220210, 1/100 dilution; anti-IL-1 $\beta$ : Abcam, Cat\#ab205924, 1/200 dilution.

Bone assessment and micro-CT analysis. The ankle joints collected before the treatment and 2 days after the last treatment in the therapeutic study were fixed in $4 \%$ paraformaldehyde and scanned at $80 \mathrm{kV}$ and $500 \mu \mathrm{A}$ with a resolution of $15 \mu \mathrm{m}$ by ex vivo micro-computed tomography (Micro-CT, VivaCT 80, SCANCO Medical AG, Switzerland). The dataset was then reconstructed to obtain the 3D images of the joints and trabecular in the distal femur. Bone morphometric parameters including BMD, bone surface vs. bone volume (BS/BV). trabecular number (Tb.N), trabecular bone thickness (Tb.Th), and trabecular separation (Tb.Sp) were also quantitatively analyzed. Ankle joints from both early and late stages AIA rats were also applied for micro-CT analysis.

Safety evaluation. To assess the in vivo safety of CEL-PRNPs, healthy male Wistar rats $(200 \pm 20 \mathrm{~g})$ were intravenously administered with $1 \mathrm{mg} / \mathrm{kg}$ of CEL equivalents of CEL solution, CEL-NPs, CEL-RNPs, or CEL-PRNPs, respectively. An equal volume of saline was injected into the control rats. Rats were sacrificed 2 days after the last treatment, and the blood and major organs (heart, liver, spleen, lung, kidney, and brain) were collected for serum enzyme and histopathological analyses. 
In addition, collected organs were sectioned for TUNEL staining and then analyzed with a laser scanning confocal microscope.

Statistical analysis. All quantitative parameters were presented as mean with standard deviation. For a two-group comparison, a Student's two-sided $t$ test was performed for the statistical analysis. For multiple comparisons, the data were analyzed using a two-way analysis of variance (ANOVA). A significant difference was considered when the $P$ value was less than 0.05 .

Reporting summary. Further information on research design is available in the Nature Research Reporting Summary linked to this article.

\section{Data availability}

All data are available in the Article, Supplementary Information files, or from the corresponding author upon reasonable request. The source data underlying Figs. 1b, $c$, 2d, 3d-g, 4b-f, 5d-f, 7c, 8b, c, and 9c-g and Supplementary Figs. 3a, b, 7b, 8b, 9b-f, 10, 12, 14a-c, 15a-d, 16, 18a-d, 19b, and 20b, c and Supplementary Table 1 are provided as a Source Data file. Source data are provided with this paper.

Received: 18 January 2020; Accepted: 5 March 2021;

Published online: 12 April 2021

\section{References}

1. Smolen, J. S. et al. Rheumatoid arthritis. Nat. Rev. Dis. Prim. 4, 18001 (2018).

2. Yeo, L. et al. Expression of chemokines CXCL4 and CXCL7 by synovial macrophages defines an early stage of rheumatoid arthritis. Ann. Rheum. Dis. 75, 763-771 (2016).

3. Bijlsma, J. W. J., Boers, M., Saag, K. G. \& Furst, D. E. Glucocorticoids in the treatment of early and late RA. Ann. Rheum. Dis. 62, 1033-1037 (2003).

4. Benito, M. J., Veale, D. J., FitzGerald, O., van den Berg, W. B. \& Bresnihan, B. Synovial tissue inflammation in early and late osteoarthritis. Ann. Rheum. Dis. 64, 1263-1267 (2005).

5. Schett, G. \& Gravallese, E. Bone erosion in rheumatoid arthritis: mechanisms, diagnosis and treatment. Nat. Rev. Rheumatol. 8, 656-664 (2012).

6. Harre, U. et al. Glycosylation of immunoglobulin G determines osteoclast differentiation and bone loss. Nat. Commun. 6, 6651 (2015).

7. Weyand, C. M. \& Goronzy, J. J. Immunometabolism in early and late stages of rheumatoid arthritis. Nat. Rev. Rheumatol. 13, 291-301 (2017).

8. Rigby, W. et al. Effect of rituximab on physical function and quality of life in patients with rheumatoid arthritis previously untreated with methotrexate. Arthritis Care Res. 63, 711-720 (2011).

9. Zhang, F. et al. Defining inflammatory cell states in rheumatoid arthritis joint synovial tissues by integrating single-cell transcriptomics and mass cytometry. Nat. Immunol. 20, 928-942 (2019).

10. Alivernini, S. et al. Distinct synovial tissue macrophage subsets regulate inflammation and remission in rheumatoid arthritis. Nat. Med. 26, 1295-1306 (2020).

11. Adamopoulos, I. E. \& Mellins, E. D. Alternative pathways of osteoclastogenesis in inflammatory arthritis. Nat. Rev. Rheumatol. 11 189-194 (2015).

12. Gough, A. et al. Osteoclastic activation is the principal mechanism leading to secondary osteoporosis in rheumatoid arthritis. J. Rheumatol. 25, 1282-1289 (1998).

13. O'Brien, W. et al. RANK-independent osteoclast formation and bone erosion in inflammatory arthritis. Arthritis Rheumatol. 68, 2889-2900 (2016).

14. Boyle, W. J., Simonet, W. S. \& Lacey, D. L. Osteoclast differentiation and activation. Nature 423, 337-342 (2003).

15. Teitelbaum, S. L. \& Ross, F. P. Genetic regulation of osteoclast development and function. Nat. Rev. Genet .4, 638-649 (2003).

16. Tong, X. et al. Suppression of AMP-activated protein kinase reverses osteoprotegerin-induced inhibition of osteoclast differentiation by reducing autophagy. Cell Prolif. 53, e12714 (2020).

17. Pöllinger, B. et al. Th17 cells, not $\mathrm{IL}_{-1} 7^{+} \gamma \delta \mathrm{T}$ cells, drive arthritic bone destruction in mice and humans. J. Immunol. 186, 2602-2612 (2011).

18. Malmström, V., Catrina, A. I. \& Klareskog, L. The immunopathogenesis of seropositive rheumatoid arthritis: from triggering to targeting. Nat. Rev. Immunol. 17, 60-75 (2017).

19. Lin, T. et al. Inhibition of osteoporosis by the $\alpha v \beta 3$ integrin antagonist of rhodostomin variants. Eur. J. Pharm. 804, 94-101 (2017).

20. Wilder, R. L. Integrin alpha $\mathrm{V}$ beta 3 as a target for treatment of rheumatoid arthritis and related rheumatic diseases. Ann. Rheum. Dis. 61, ii96-iig9 (2002).

21. Pope, R. M. Apoptosis as a therapeutic tool in rheumatoid arthritis. Nat. Rev. Immunol. 2, 527-535 (2002).
22. Hao, S. et al. Critical role of CCL22/CCR4 axis in the maintenance of immune homeostasis during apoptotic cell clearance by splenic CD8a+ CD103+ dendritic cells. Immunology 148, 174-186 (2016).

23. Luo, J. et al. LGR4 is a receptor for RANKL and negatively regulates osteoclast differentiation and bone resorption. Nat. Med. 22, 539-546 (2016).

24. Han, Y., You, X., Xing, W., Zhang, Z. \& Zou, W. Paracrine and endocrine actions of bone-the functions of secretory proteins from osteoblasts, osteocytes, and osteoclasts. Bone Res. 6, 16 (2018).

25. $\mathrm{Xu}, \mathrm{K}$. et al. Reduced apoptosis correlates with enhanced autophagy in synovial tissues of rheumatoid arthritis. Inflamm. Res. 62, 229-237 (2013).

26. Zhang, Q. et al. Tumor necrosis factor prevents alendronate-induced osteoclast apoptosis in vivo by stimulating Bcl-xL expression through Ets-2. Arthritis Rheum. 52, 2708-2718 (2005).

27. Døhn, U. M. et al. No overall progression and occasional repair of erosions despite persistent inflammation in adalimumab-treated rheumatoid arthritis patients: results from a longitudinal comparative MRI, ultrasonography, CT and radiography study. Ann. Rheum. Dis. 70, 252-258 (2011).

28. Moghadam-Kia, S. \& Werth, V. P. Prevention and treatment of systemic glucocorticoid side effects. Int. J. Dermatol. 49, 239-248 (2010).

29. Patschan, D., Loddenkemper, K. \& Buttgereit, F. Molecular mechanisms of glucocorticoid-induced osteoporosis. Bone 29, 498-505 (2001).

30. Dinarello, C. A. Anti-inflammatory agents: present and future. Cell 140, 935-950 (2010).

31. Nishimoto, N. et al. Long-term safety and efficacy of tocilizumab, an anti-IL-6 receptor monoclonal antibody, in monotherapy, in patients with rheumatoid arthritis (the STREAM study): evidence of safety and efficacy in a 5-year extension study. Ann. Rheum. Dis. 68, 1580-1584 (2009).

32. Nishimoto, N. et al. Study of active controlled monotherapy used for rheumatoid arthritis, an IL-6 inhibitor (SAMURAI): evidence of clinical and radiographic benefit from an $\mathrm{x}$ ray reader-blinded randomised controlled trial of tocilizumab. Ann. Rheum. Dis. 66, 1162-1167 (2007)

33. Winthrop, K. L. The emerging safety profile of JAK inhibitors in rheumatic disease. Nat. Rev. Rheumatol. 13, 234-243 (2017).

34. Vyas, D., O'Dell, K. M., Bandy, J. L. \& Boyce, E. G. Tofacitinib: the First Janus Kinase (JAK) inhibitor for the treatment of rheumatoid arthritis. Ann. Pharmacother. 47, 1524-1531 (2013).

35. Kremer, J. M. et al. A phase IIb dose-ranging study of the oral JAK inhibitor tofacitinib (CP-690,550) versus placebo in combination with background methotrexate in patients with active rheumatoid arthritis and an inadequate response to methotrexate alone. Arthritis Rheumatol. 64, 970-981 (2014).

36. Sethi, G., Ahn, K. S., Pandey, M. K. \& Aggarwal, B. B. Celastrol, a novel triterpene, potentiates TNF-induced apoptosis and suppresses invasion of tumor cells by inhibiting NF- $\mathrm{kB}-$ regulated gene products and TAK1-mediated NF- $\mathrm{kB}$ activation. Blood 109, 2727-2735 (2006).

37. Pang, $\mathrm{X}$. et al. Celastrol suppresses angiogenesis-mediated tumor growth through inhibition of AKT/mammalian target of rapamycin pathway. Cancer Res. 70, 1951-1959 (2010).

38. Guo, L. et al. Targeted delivery of celastrol to mesangial cells is effective against mesangioproliferative glomerulonephritis. Nat. Commun. 8, 878 (2017).

39. Louis, C. et al. Therapeutic effects of a TANK-binding kinase 1 inhibitor in germinal center-driven collagen-induced arthritis. Arthritis Rheumatol. 71, 50-62 (2019).

40. Szekanecz, Z. \& Koch, A. E. Successes and failures of chemokine-pathway targeting in rheumatoid arthritis. Nat. Rev. Rheumatol. 12, 5-13 (2015).

41. Courbon, G., Cleret, D., Linossier, M., Vico, L. \& Marotte, H. Early subchondral bone loss at arthritis onset predicted late arthritis severity in a rat arthritis model. J. Cell Physiol. 232, 1318-1325 (2017).

42. Norling, L. V. et al. Proresolving and cartilage-protective actions of resolvin D1 in inflammatory arthritis. JCI Insight 1, e85922 (2016).

43. Zhang, Q. et al. Neutrophil membrane-coated nanoparticles inhibit synovial inflammation and alleviate joint damage in inflammatory arthritis. Nat. Nanotechnol. 13, 1182-1190 (2018).

44. van der Voort, R. et al. Elevated CXCL16 expression by synovial macrophages recruits memory T cells into rheumatoid joints. Arthritis Rheum. 52, 1381-1391 (2005).

45. Thenappan, T. et al. A central role for $\mathrm{CD} 68(+)$ macrophages in hepatopulmonary syndrome: reversal by macrophage depletion. Am. J. Respir. Crit. Care Med. 183, 1080-1091 (2011).

46. Mucke, J. et al. Inhomogeneity of immune cell composition in the synovial sublining: linear mixed modelling indicates differences in distribution and spatial decline of CD68+ macrophages in osteoarthritis and rheumatoid arthritis. Arthritis Res. Ther. 18, 170 (2016).

47. Kim, W. et al. LIGHT is involved in the pathogenesis of rheumatoid arthritis by inducing the expression of pro-inflammatory cytokines and MMP-9 in macrophages. Immunology 114, 272-279 (2005).

48. Krumbholz, G. et al. Response of human rheumatoid arthritis osteoblasts and osteoclasts to adiponectin. Clin. Exp. Rheumatol. 35, 406-414 (2017). 
49. Fredman, G. et al. Targeted nanoparticles containing the proresolving peptide Ac2-26 protect against advanced atherosclerosis in hypercholesterolemic mice. Sci. Transl. Med. 7, 275ra220-275ra220 (2015).

50. Liu, J., Zhang, R. \& Xu, Z. P. Nanoparticle-based nanomedicines to promote cancer immunotherapy: recent advances and future directions. Small 15, 1900262 (2019).

51. $\mathrm{Xu}, \mathrm{Q}$. et al. Impact of surface polyethylene glycol (PEG) density on biodegradable nanoparticle transport in mucus ex Vivo and distribution in Vivo. ACS Nano 9, 9217-9227 (2015).

52. Zhang, Q. et al. A safe and efficient hepatocyte-selective carrier system based on myristoylated preS1/21-47 domain of hepatitis B virus. Nanoscale 7, 9298-9310 (2015).

53. Dou, C. et al. Graphene-based microRNA transfection blocks preosteoclast fusion to increase bone formation and vascularization. Adv. Sci. 5, 1700578 (2018).

54. Hock, J. M. et al. Osteoblast apoptosis and bone turnover. J. Bone Min. Res. 16, 975-984 (2001).

55. Li, J. et al. Treatment of arthritis by macrophage depletion and immunomodulation: testing an apoptosis-mediated therapy in a humanized death receptor mouse model. Arthritis Rheum. 64, 1098-1109 (2012).

56. Kroemer, G., Galluzzi, L. \& Brenner, C. Mitochondrial membrane permeabilization in cell death. Physiol. Rev. 87, 99-163 (2007).

57. Chen, W. et al. Black phosphorus nanosheets as a neuroprotective nanomedicine for neurodegenerative disorder therapy. Adv. Mater. 30 , 1703458 (2018).

58. Singh, B., Fu, C. \& Bhattacharya, J. Vascular expression of the alpha(v)beta(3)integrin in lung and other organs. Am. J. Physiol. Lung Cell Mol. Physiol. 278, L217-L226 (2000).

59. Liu, S. et al. 18F-labeled galacto and PEGylated RGD dimers for PET imaging of av $\beta 3$ integrin expression. Mol. Imaging Biol. 12, 530-538 (2010).

60. Walsh, N. C. \& Bone, E. M. G. remodeling in rheumatic disease: a question of balance. Immunol. Rev. 233, 301-312 (2010).

61. Inoue, K., Hu, X. \& Zhao, B. Regulatory network mediated by RBP-J/NFATc1miR182 controls inflammatory bone resorption. FASEB J. 34, 2392-2407 (2020).

62. Zhao, J. et al. MiR-146a deletion protects from bone loss in OVX mice by suppressing RANKL/OPG and M-CSF in bone microenvironment. J. Bone Min. Res 34, 2149-2161 (2019).

63. Kostenuik, P. J. Osteoprotegerin and RANKL regulate bone resorption, density, geometry and strength. Curr. Opin. Pharm. 5, 618-625 (2005).

64. van Tuyl, L. H. D. et al. Baseline RANKL:OPG ratio and markers of bone and cartilage degradation predict annual radiological progression over 11 years in rheumatoid arthritis. Ann. Rheum. Dis. 69, 1623 (2010).

65. Firestein, G. S. Evolving concepts of rheumatoid arthritis. Nature 423, 356-361 (2003).

66. Kinne, R. W., Bräuer, R., Stuhlmüller, B., Palombo-Kinne, E. \& Burmester, G.R. Macrophages in rheumatoid arthritis. Arthritis Res. Ther. 2, 189-202 (2000).

67. Paoletti, A. et al. Monocyte/macrophage abnormalities specific to rheumatoid arthritis are linked to miR-155 and are differentially modulated by different TNF inhibitors. J. Immunol. 203, 1766-1775 (2019).

68. Ulukaya, E., Acilan, C. \& Yilmaz, Y. Apoptosis: why and how does it occur in biology? Cell Biochem. Funct. 29, 468-480 (2011).

69. Weiss, G. \& Schaible, U. E. Macrophage defense mechanisms against intracellular bacteria. Immunol. Rev. 264, 182-203 (2015).

70. Westphalen, K. et al. Sessile alveolar macrophages communicate with alveolar epithelium to modulate immunity. Nature 506, 503-506 (2014).

71. Zheng, L. et al. Simultaneous NF- $\mathrm{kB}$ inhibition and E-cadherin upregulation mediate mutually synergistic anticancer activity of celastrol and SAHA in vitro and in vivo. Int. J. Cancer 135, 1721-1732 (2014).

72. Smolen, J. S. \& Steiner, G. Therapeutic strategies for rheumatoid arthritis. Nat. Rev. Drug Discov. 2, 473-488 (2003).

73. Wang, Y. et al. Enhanced therapeutic effect of RGD-modified polymeric micelles loaded with low-dose methotrexate and nimesulide on rheumatoid arthritis. Theranostics 9, 708-720 (2019).

74. Santos Savio, A. et al. Differential expression of pro-inflammatory cytokines IL-15Ralpha, IL-15, IL-6 and TNFalpha in synovial fluid from rheumatoid arthritis patients. BMC Musculoskel. Dis. 16, 51 (2015).
75. Liu, H. et al. Regulation of Mcl-1 expression in rheumatoid arthritis synovial macrophages. Arthritis Rheum. 54, 3174-3181 (2006).

76. Meng, J. et al. Catalpol suppresses osteoclastogenesis and attenuates osteoclast-derived bone resorption by modulating PTEN activity. Biochem. Pharm. 171, 113715 (2020)

77. Nishikawa, K. et al. Blimp1-mediated repression of negative regulators is required for osteoclast differentiation. Proc. Natl Acad. Sci. USA 107, 3117-3122 (2010)

78. Han, S. A., Lee, S., Seong, S. C. \& Lee, M. C. Effects of CD14 macrophages and proinflammatory cytokines on chondrogenesis in osteoarthritic synoviumderived stem cells. Tissue Eng. Part A 20, 2680-2691 (2014).

79. Tu, J. et al. Synovial macrophages in rheumatoid arthritis: the past, present, and future. Mediat. Inflamm. 4, 1-8 (2020)

80. Wang, Q. et al. Targeted delivery of low-dose dexamethasone using PCL-PEG micelles for effective treatment of rheumatoid arthritis. J. Control Release 230, 64-72 (2016).

\section{Acknowledgements}

This work was financially supported by the National Natural Science Foundation of China (No. 81872804) and Sichuan major science and technology project on biotechnology and medicine (2018SZDZX0018). In addition, the authors would like to thank Dr. Li Chen from Analytical \& Testing Center Sichuan University for her help with micro-CT scanning and analysis.

\section{Author contributions}

T.G. and G.L. conceived and planned the study. C.D. carried out the experiments, generated and analyzed data, created Figs. 2a and 8a and Supplementary Fig. 20a, and wrote the original paper. Q.Z., P.H., and K.H. helped with animal and cell studies. B.Z collected human samples and helped with related experiments. X.S., T.G., and Z.Z. helped with paper editing.

\section{Competing interests}

The authors declare no competing interests.

\section{Additional information}

Supplementary information The online version contains supplementary material available at https://doi.org/10.1038/s41467-021-22454-z.

Correspondence and requests for materials should be addressed to G.L. or T.G.

Peer review information Nature Communications thanks Joaquim Oliveira and the other, anonymous reviewer(s) for their contribution to the peer review of this work. Peer review reports are available.

Reprints and permission information is available at http://www.nature.com/reprints

Publisher's note Springer Nature remains neutral with regard to jurisdictional claims in published maps and institutional affiliations.

Open Access This article is licensed under a Creative Commons Attribution 4.0 International License, which permits use, sharing, adaptation, distribution and reproduction in any medium or format, as long as you give appropriate credit to the original author(s) and the source, provide a link to the Creative Commons license, and indicate if changes were made. The images or other third party material in this article are included in the article's Creative Commons license, unless indicated otherwise in a credit line to the material. If material is not included in the article's Creative Commons license and your intended use is not permitted by statutory regulation or exceeds the permitted use, you will need to obtain permission directly from the copyright holder. To view a copy of this license, visit http://creativecommons.org/ licenses/by/4.0/

(C) The Author(s) 2021 